\title{
Influence of the single-particle structure on the nuclear surface and the neutron skin
}

\author{
M. Warda, ${ }^{1, *}$ M. Centelles, ${ }^{2, \dagger}$ X. Viñas, ${ }^{2, \ddagger}$ and X. Roca-Maza ${ }^{3, \S}$ \\ ${ }^{1}$ Katedra Fizyki Teoretycznej, Uniwersytet Marii Curie-Skłodowskiej, ul. Radziszewskiego 10, 20-031 Lublin, Poland \\ ${ }^{2}$ Departament d'Estructura i Constituents de la Matèria and Institut de Ciències del Cosmos (ICC), Facultat de Física, \\ Universitat de Barcelona, Diagonal 645, E-08028 Barcelona, Spain \\ ${ }^{3}$ Dipartimento di Fisica, Università degli Studi di Milano and INFN, Sezione di Milano, via Celoria 16, I-20133 Milano, Italy
}

(Received 3 April 2014; published 4 June 2014)

\begin{abstract}
We analyze the influence of the single-particle structure on the neutron density distribution and the neutron skin in $\mathrm{Ca}, \mathrm{Ni}, \mathrm{Zr}, \mathrm{Sn}$, and $\mathrm{Pb}$ isotopes. The nucleon density distributions are calculated in the Hartree-Fock+BCS approach with the SLy4 Skyrme force. A close correlation is found between the quantum numbers of the valence neutrons and the changes in the position and the diffuseness of the nuclear surface, which in turn affect the neutron skin thickness. Neutrons in the valence orbitals with low principal quantum number and high angular momentum mainly displace the position of the neutron surface outwards, while neutrons with high principal quantum number and low angular momentum basically increase the diffuseness of the neutron surface. The impact of the valence shell neutrons on the tail of the neutron density distribution is discussed.
\end{abstract}

DOI: 10.1103/PhysRevC.89.064302

PACS number(s): 21.10.Gv, 21.60.Jz

\section{INTRODUCTION}

The spatial distribution of nucleons inside a nucleus is one of the most basic topics in nuclear physics. The density distribution of protons is quite well mapped from the numerous experiments of elastic electron-nucleus and muon-nucleus scattering $[1,2]$ and the charge radii of many nuclei are known with uncertainties well below $1 \%$ [3]. Neutrons, as neutral particles, are much harder to resolve, and knowledge about their spatial layout in a nucleus is still limited. Until now, neutron radii have been measured in less than thirty isotopes, and the experimental neutron density distribution is known only in a few nuclei with relatively large error bars [4-12]. Thus, information about the layout of neutrons inside a nucleus often comes only from the theoretical predictions at present. However, new experimental advances in techniques such as elastic proton scattering [12] and coherent pion photoproduction from nuclei [13,14], and the advent of parityviolating elastic electron scattering facilities [15-17], suggest that largely improved determinations of neutron radii and neutron density distributions may be possible in the near future.

Knowledge of neutron distributions is very important, as it constitutes a necessary input in a wide range of problems in physics. It is strongly related to the isospin properties of nuclear forces and the nuclear symmetry energy [18-23]. The profile of the neutron density distribution is demanded as an input in the analysis of many scattering experiments. The arrangement of neutrons in nuclei is important for collective nuclear excitations [24,25], such as giant dipole resonance [26,27] and pygmy dipole resonance [28-30]. Precise knowledge of the neutron skin thickness (NST), i.e., the difference between the neutron and proton root mean square

\footnotetext{
*warda@kft.umcs.lublin.pl

†mariocentelles@ub.edu

${ }^{\ddagger}$ xavier@ecm.ub.edu

§xavier.roca.maza@mi.infn.it
}

(rms) radii,

$$
\Delta r_{n p}=\left\langle r^{2}\right\rangle_{n}^{1 / 2}-\left\langle r^{2}\right\rangle_{p}^{1 / 2},
$$

is not only of interest in nuclear structure physics. This quantity is strongly correlated, within the realm of nuclear mean-field theories [18,31-38], with the slope of the nuclear symmetry energy at saturation density, and therefore may be used to constrain the equation of state of neutron-rich matter. Thus, the results of the investigation of the distribution of neutrons in atomic nuclei affect studies of such distant areas of physics as heavy-ion collisions [39-42], scattering of polarized electrons on nuclei [15,36,43-47], precision tests of the standard model by atomic parity violation [48,49], and nuclear astrophysics [50-54].

Theoretical predictions of neutron density distributions can be verified, in principle, by the comparison of the calculated values of the neutron rms radii and of the NST with the available experimental data. The uncertainties in the measured values leave much freedom for theoretical neutron density distributions $[8,9,55,56]$. Moreover, rms radii and the NST are general properties of the neutron density distribution; different density profiles (e.g., those calculated with various nuclear forces) may give the same values of the neutron rms radius and the NST [57]. Therefore, careful theoretical studies are required to understand the physics of nucleon distributions inside a nucleus. The main issues affecting the deviations of the NST from the picture of a smooth variation with the neutron excess of the nucleus are nuclear deformation [55] and the quantum-mechanical properties of the nucleonic orbitals. In the present article we concentrate on the latter effect.

The basic features of the neutron skin of nuclei can be explained by the nuclear droplet model (DM) [58,59]. The DM predicts that the NST grows on average linearly with the relative neutron excess $I=(N-Z) / A$, which was confirmed by the experimental data of Refs. [8,9]. In the standard version of the DM the neutron and proton surface diffusenesses are assumed to be equal, although the influence of different surface diffusenesses between neutrons and 
protons was also investigated $[19,59,60]$. In particular, it has been shown that the surface contribution to the NST in nuclear mean-field models is not negligible [19,35,57,60,61]; indeed, self-consistent mean-field calculations predict in many neutron-rich isotopes twice thicker neutron than proton surface diffuseness. Moreover, abrupt changes of the surface diffuseness between isotopes may appear (see Figs. 7 and 8 of Ref. [57]). It has been found that the surface of the neutron distribution is narrower in the doubly magic nuclei, whereas it extends over a wider region in the midshell nuclei. Such a behavior suggests that quantum shell effects impinge on the NST on top of the macroscopic DM predictions.

There is significant interest currently in the exploration of the properties of exotic nuclei, as the radioactive ion beam facilities in laboratories worldwide are extending the nuclear landscape to new limits. We have devoted some effort in previous works $[62,63]$ to study the evolution of the nuclear charge density from stable to exotic nuclei, and its relation with the changes of the proton shell structure in isotopic and isotonic chains. In the present article we investigate the influence of the single-particle $(\mathrm{sp})$ properties of the valence neutrons on the nuclear surface and the neutron skin of stable and unstable nuclei. We study several isotopic chains representative of different mass regions in order to examine the changes of the neutron skin when subsequent neutrons are added into particular orbitals. We analyze two mechanisms of generating the neutron skin. One of them arises from a displacement between the positions of the equivalent neutron and proton sharp surfaces (it is mostly a "bulk" effect). The other one is a consequence of different surface diffuseness between the neutron and proton density profiles (it is mostly a "surface" effect). To minimize the influence of deformation on the results [55] it is helpful to choose nuclei with magic proton number, which are mostly spherical nuclei in their ground states. Hence, we take into consideration the $\mathrm{Ca}, \mathrm{Ni}, \mathrm{Zr}, \mathrm{Sn}$, and $\mathrm{Pb}$ elements. We focus our study mainly on the neutron-rich nuclei because the neutron skin is larger and more sensitive to isotopic effects there. We concentrate on the last full major shell (or shells) of the considered elements. In this way we examine the neutron skin in $\mathrm{Ca}$ and $\mathrm{Ni}$ from $N=20$ to $N=50$, in $\mathrm{Zr}$ and $\mathrm{Sn}$ from $N=50$ to $N=82$, in Sn between $N=82$ and $N=126$, and in $\mathrm{Pb}$ from $N=126$ to $N=184$. We start with the analysis of the $\mathrm{Sn}$ isotopes ranging from $N=82$ to 126 because the discussed sp properties are quite well magnified and easy to describe in this relatively long chain. After this illustrative example we study the other elements.

The structure of this article is the following. In Sec. II the basic ideas of our theoretical description of the NST are collected (an extended presentation can be found in Refs. [35,57]). The detailed analysis of the results for the NST in the $\mathrm{Sn}$ isotopes is presented in Sec. III. The other isotopic chains are discussed in Sec. IV. Finally, the conclusions are presented in Sec. V.

\section{BULK AND SURFACE CONTRIBUTIONS TO THE NEUTRON SKIN THICKNESS}

In our study we compute the density distributions of nucleons in even-even isotopes in self-consistent Hartree-Fock calculations using the SLy4 nuclear functional [64]. SLy4 is a Skryme-type force that was developed with a focus on neutron-rich nuclei and the equation of state of neutron matter. It has been successfully applied in studies of a wide range of phenomena, and it is known to describe reasonably well nuclear properties such as masses, deformations, nucleon separation energies, and radii along the periodic table [65]. Though the general features of the NST described in this paper are basically independent of the nuclear mean-field interaction used to compute them, the fine details can depend to a certain extent on the nuclear interaction. In our calculations we assume spherical symmetry for all considered nuclei (however, some of the $\mathrm{Zr}$ isotopes are known to be deformed in their ground state). We use a volume BCS pairing with pairing strengths $V_{0}^{n}=300 \mathrm{MeV}$ and $V_{0}^{p}=360 \mathrm{MeV}$. The pairing window is taken to be $1 \hbar \omega$ above the Fermi level. To fit the pairing strengths we used as reference data the SLy4 results of the HFB+Lipkin-Nogami model of Ref. [66] for the binding energies of the midshell nuclei ${ }^{116} \mathrm{Sn}$ for neutron pairing and ${ }^{144} \mathrm{Sm}$ for proton pairing. The treatment of the continuum for neutron-rich nuclei close to the drip line is done following the prescription given in Ref. [67]. In this way the HFB energies reported in Ref. [66] are overall well reproduced from the proton to the neutron drip line. We find the two-neutron drip line of the elements considered in the present work at ${ }^{68} \mathrm{Ca}$, ${ }^{78} \mathrm{Ni},{ }^{122} \mathrm{Zr}$, ${ }^{176} \mathrm{Sn}$, and ${ }^{266} \mathrm{~Pb}$, which is the same result as in Ref. [66] excepting that the drip line nucleus ${ }^{174} \mathrm{Sn}$ of Ref. [66] is shifted to ${ }^{176} \mathrm{Sn}$ in our calculation.

To get a better grasp of the properties of the neutron skin it can be useful to fit the density profiles obtained in the self-consistent mean-field calculations by two-parameter Fermi $(2 \mathrm{pF})$ distributions [2,68]:

$$
\rho(r)=\frac{\rho_{0}}{1+\exp [(r-C) / a]},
$$

where $\rho_{0}$ is the central density, $C$ is the half-density radius, and $a$ describes the surface diffuseness. As a result, one obtains numerically the two most important quantities characterizing the shape of the density profiles: namely, the position and the thickness of the nuclear surface. Both of them are crucial for a proper determination of the NST, as the NST is defined through the rms radii and consequently it is very sensitive to the density profile at the surface. There is no unique prescription to parametrize a given density profile with a $2 \mathrm{pF}$ function. Following earlier works [35,57], we fit the $a, C$, and $\rho_{0}$ parameters to reproduce the quadratic and quartic moments of the neutron or proton density distribution, and the number of nucleons. It has been shown that this method reproduces with good accuracy the surface region of any realistic density profile given as an input [35,57].

The neutron skin can be easily understood assuming $2 \mathrm{pF}$ distributions for both neutrons and protons. Indeed, Fermi-type densities are common in the extraction of neutron skins from different experiments, as in the case of neutron skins deduced from exotic atoms $[8,69,70]$ or from coherent pion photoproduction cross sections $[13,14]$. Within the context of $2 \mathrm{pF}$ densities, it has become popular to discern two main scenarios for the neutron skin of nuclei. In the first scenario, the neutron skin is formed when the neutron half-density 
radius is larger than the proton half-density radius, and the surface diffusenesses of neutrons and protons are the same (i.e., $C_{n}>C_{p}$ and $a_{n}=a_{p}$ ). Such $2 \mathrm{pF}$ density profiles are called a "skin" type distribution [8]. The alternative scenario assumes that the differences between both density profiles are due to an enlarged neutron surface diffuseness with the same neutron and proton half-density radii (i.e., $a_{n}>a_{p}$ and $\left.C_{n}=C_{p}\right)$. The corresponding $2 \mathrm{pF}$ density profiles are called a "halo" type distribution [8]. In general, both situations may coexist in a nucleus, simultaneously contributing to the NST [18,19,35,57,59].

Despite the simplicity of describing the density profiles through $2 \mathrm{pF}$ distributions, we have shown in previous papers $[35,57]$ that the half-density radii $C_{n}$ and $C_{p}$ are not the most appropriate radii for extracting the bulk and surface contributions to the NST. Following Refs. $[35,57]$ we introduce the bulk contribution as

$$
\Delta r_{n p}^{\mathrm{bulk}} \equiv \sqrt{\frac{3}{5}}\left(R_{n}-R_{p}\right),
$$

where $R_{n}$ and $R_{p}$ are the neutron and proton equivalent sharp radii, respectively [68]. The equivalent sharp radius $R$ corresponds to a sharp distribution with a uniform density, equal to the bulk value of the actual density, having the same number of particles [68]. As can be seen in Fig. 1 of Ref. [35] and Fig. 2 of Ref. [57] (see also Ref. [68]), a sharp sphere with radius $C$ overestimates the original mean-field density in the whole nuclear interior, whereas a sharp density distribution with radius $R$ is able to reproduce properly the bulk part of the original density profile. Therefore, the sharp radius $R$ rather than the half-density radius $C$ is the suitable radius to describe the size of the bulk region of the nucleus $[35,57,68]$.

It is possible to express $R$ in terms of the parameters $C$ and $a$ of the $2 \mathrm{pF}$ distributions [35,57,68], so that the bulk contribution to the NST given by Eq. (3) can be written also as

$$
\Delta r_{n p}^{\mathrm{bulk}} \simeq \sqrt{\frac{3}{5}}\left[\left(C_{n}-C_{p}\right)+\frac{\pi^{2}}{3}\left(\frac{a_{n}^{2}}{C_{n}}-\frac{a_{p}^{2}}{C_{p}}\right)\right] .
$$

The remaining part of the NST is the surface contribution:

$$
\Delta r_{n p}^{\text {surf }} \simeq \sqrt{\frac{3}{5}} \frac{5 \pi^{2}}{6}\left(\frac{a_{n}^{2}}{C_{n}}-\frac{a_{p}^{2}}{C_{p}}\right) .
$$

From Eqs. (4) and (5), it is clear that the difference between the half-density radii $C_{n}$ and $C_{p}$ can affect not only the bulk contribution but also the surface contribution to the NST. Similarly, the difference between the surface diffusenesses $a_{n}$ and $a_{p}$ of the $2 \mathrm{pF}$ profiles affects $\Delta r_{n p}^{\text {surf }}$ and also $\Delta r_{n p}^{\text {bulk }}$. In general, both the changes of the half-density radii and of the surface diffusenesses contribute simultaneously to the NST.

Although the measurements provide data of the total NST only, from the theoretical point of view it is interesting to analyze the separate contributions of the bulk and surface parts of the NST. Useful information about the nuclear surface can be obtained from such investigation. In the next sections we analyze how the bulk and the surface contributions change along the selected isotopic chains and how they are correlated with the sp properties of the valence neutrons.

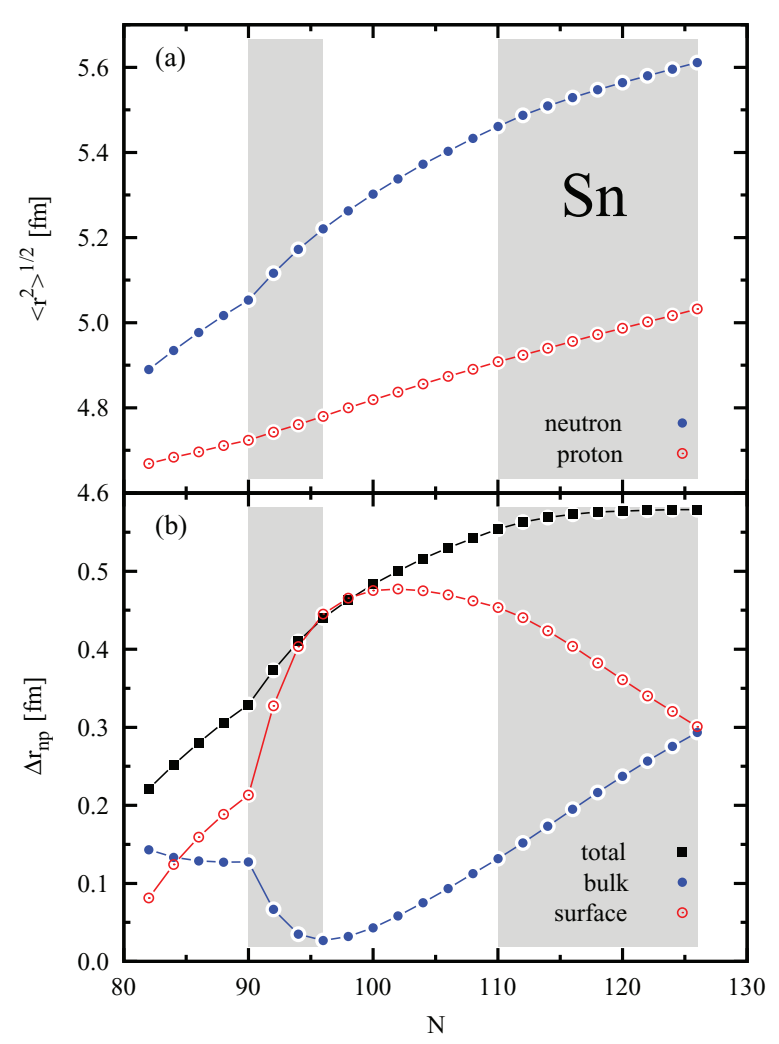

FIG. 1. (Color online) (a) Neutron and proton rms radii in $\mathrm{Sn}$ isotopes with $82 \leqslant N \leqslant 126$ calculated with the SLy4 Skyrme force. (b) The NST of the Sn isotopes (black squares), and the corresponding bulk (blue dots) and surface (red circles) contributions to the NST [Eqs. (3) and (5)].

\section{NEUTRON SKIN IN THE Sn ISOTOPIC CHAIN FOR $82 \leqslant N \leqslant 126$}

The NST grows along the isotopic chain of a given element. The DM of Myers and Świątecki [58,71,72] explains this behavior as it predicts a faster linear increase with the relative neutron excess $I=(N-Z) / A$ for the neutron rms radius than for the proton rms radius. The rate of increase of the NST with $I$ is related to the density dependence of the nuclear symmetry energy $[18,19]$. Calculations with nuclear mean field models show that deviations from the linear growth with $I$ can be found in the NST [57]. It is easy to show that these deviations are connected with shell properties of nuclei. Indeed, the NST displays local minima for magic neutron numbers, whereas it exceeds the average trend for midshell isotopes (see Fig. 9 of Ref. [57]). Therefore, to explain this nonlinearity, we have to investigate the sp structure of nuclei. As a first example, we study neutron-rich $\mathrm{Sn}$ isotopes of the major shell ranging from $N=82$ to $N=126$. The last isotope in this chain is the drip-line nucleus.

\section{A. Correlation of neutron skin properties with quantum numbers of valence neutrons}

In Fig. 1(a) it is easy to see that both the neutron and the proton rms radii of $\mathrm{Sn}$ increase with increasing neutron number, and that the slope for neutrons is larger than for 
protons, as predicted by the DM. The difference between the two curves of Fig. 1(a) is just the NST plotted in panel (b) of this figure. The proton radii show a rather linear dependence on the neutron number, whereas some departure from linearity is observed in the neutron radii. The same departure from linearity is therefore observed in the NST of the Sn isotopes. Similar properties have been found in Ref. [73] where an alternative splitting of the mean square radius into geometrical and Helm radii is applied.

The bulk (3) and the surface (5) parts of the NST are also plotted in Fig. 1(b). The lack of linearity visible in the NST diagram is magnified in the individual plots of these contributions. Four intervals of neutron number, marked in Fig. 1 by white and grey stripes, can be easily distinguished in this plot. The first interval covers the region from $N=82$ to $N=90$ where the bulk part $\Delta r_{n p}^{\text {bulk }}$ remains almost constant and the surface part $\Delta r_{n p}^{\text {surf }}$ increases roughly linearly. The second region, up to $N=96$, is characterized by a fast increase of $\Delta r_{n p}^{\text {surf }}$ and, simultaneously, a decrease of $\Delta r_{n p}^{\text {bulk }}$. Next, up to $N=110, \Delta r_{n p}^{\text {surf }}$ remains roughly constant around its maximal value reached at $N=102$ and $\Delta r_{n p}^{\text {bulk }}$ rises almost linearly with $N$. Finally, till the shell is completely filled up at the magic number $N=126, \Delta r_{n p}^{\text {bulk }}$ grows and $\Delta r_{n p}^{\text {surf }}$ decreases, both of them linearly.

In order to explain the variations of the bulk and the surface contributions in the NST diagram, selected sp properties of the considered $\mathrm{Sn}$ isotopes are plotted in Fig. 2. First, the sp energies $\varepsilon_{n l j}$ of the levels belonging to the major shell are shown in panel (a) and their occupancy $v_{n l j}^{2}$ in panel (b). The energy and the occupancy of these levels show a distinct behavior in each interval outlined above. In the first region, from $N=82$ up to $N=90$, the lowest $2 f_{7 / 2}$ level is progressively populated with neutrons. Its occupancy grows from 0 to almost 1 while the other orbitals remain unoccupied due to the large energy gap, fairly over $1 \mathrm{MeV}$, that separates the $2 f_{7 / 2}$ level from the higher levels. The $3 p_{3 / 2}$ level crosses the Fermi level between $N=90$ and $N=96$. Its occupancy increases faster in this region than the occupancy of the $1 h_{9 / 2}$, $2 f_{5 / 2}$, and $3 p_{1 / 2}$ orbitals which are slightly higher in energy. Next, from $N=96$ to $N=110$, the four aforementioned levels are filled up when more neutrons are added to the $\mathrm{Sn}$ nuclei. At $N=110$ the occupancy of these orbitals ranges between 0.7 and 0.9 and in the heaviest isotopes they are almost fully occupied. The $1 i_{13 / 2}$ level, the highest in energy, behaves otherwise. Its occupancy grows slowly up to 0.2 at $N=110$ and then it rises much faster in the heavier isotopes, without a plateau at the shell closure at $N=126$.

We can correlate the behavior of $\Delta r_{n p}^{\text {bulk }}$ and $\Delta r_{n p}^{\text {surf }}$ with the principal quantum number $n$ and the orbital angular momentum $l$ of the orbitals occupied by the valence neutrons in the intervals shown in Fig. 1(b). The type of orbitals lying in the vicinity of the Fermi level plays a crucial role in the determination of the neutron radii. When high- $n-$ low- $l$ levels within the shell are populated, one observes an increase of $\Delta r_{n p}^{\text {surf }}$, whereas low- $n$-high- $l$ levels are more correlated with the growth of $\Delta r_{n p}^{\text {bulk }}$.

In the considered shell of the $\mathrm{Sn}$ isotopes, the two $3 p$ levels (displayed with red circles in Fig. 2) have an especially

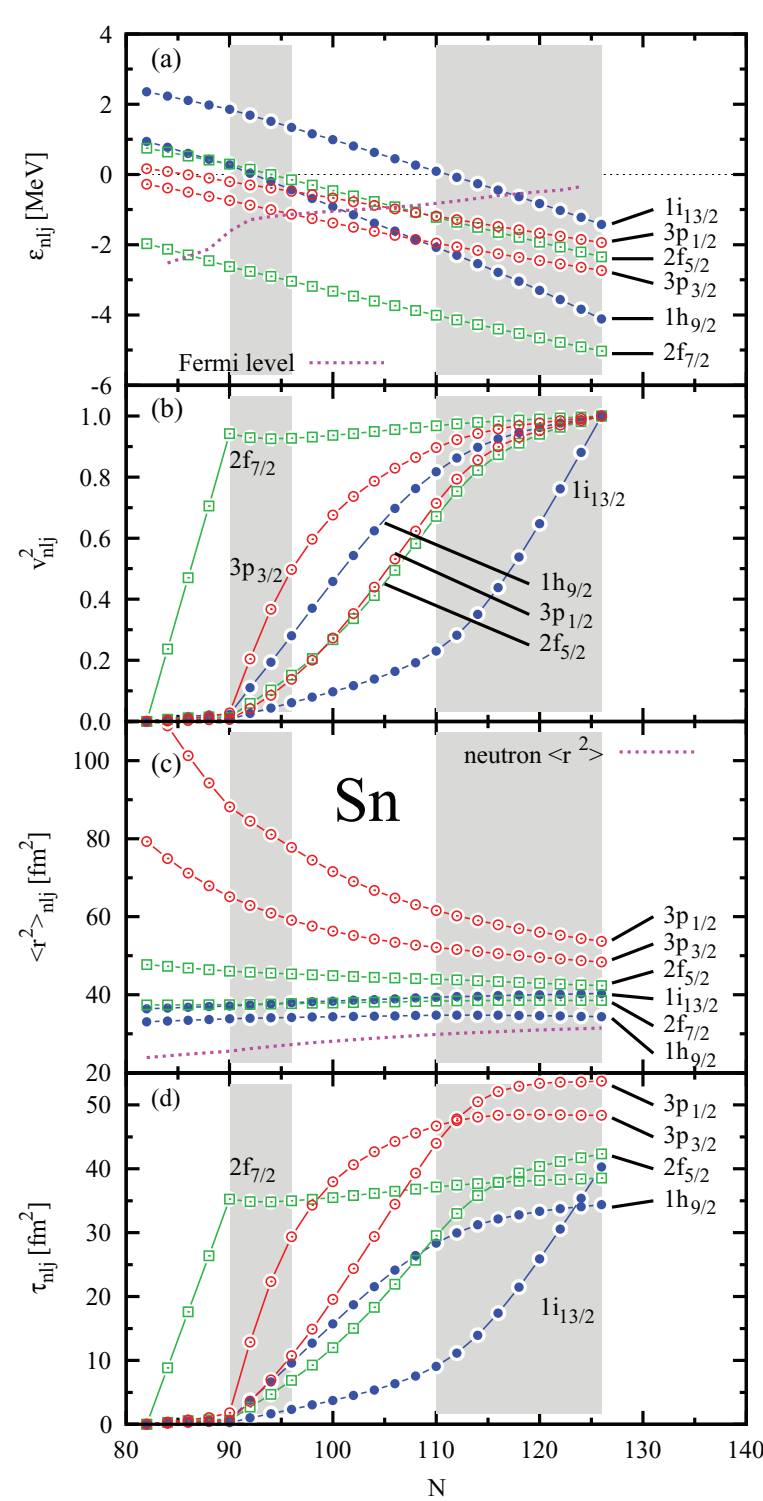

FIG. 2. (Color online) Single-particle properties of the neutron orbitals belonging to the major valence shell for $\mathrm{Sn}$ isotopes with $82 \leqslant N \leqslant 126$ calculated with the SLy4 Skyrme force. (a) Singleparticle energies and Fermi level, (b) occupancy of each orbital, (c) mean square radii of each orbital defined in Eq. (6) and total neutron mean square radius of the isotope, and (d) contributions to the mean square radius of each level $\tau_{n l j}$ defined in Eq. (7). The $3 p_{3 / 2}$ and $3 p_{1 / 2}$ orbitals are marked by red circles, the $2 f_{7 / 2}$ and $2 f_{5 / 2}$ orbitals by green squares, and the $1 h_{9 / 2}$ and $1 i_{13 / 2}$ orbitals by blue dots.

large impact on the results. At first look, it seems unexpected because only 6 neutrons out of 44 from the whole shell can occupy these two levels. To understand this effect, let us look at panel (c) of Fig. 2 where the mean square radius of each sp orbital, defined as

$$
\left\langle r^{2}\right\rangle_{n l j}=\int d \mathbf{r} r^{2} \varphi_{n l j}^{2}(\mathbf{r}),
$$

is plotted for the valence shell neutrons. In Eq. (6), $\varphi_{n l j}(\mathbf{r})$ is the normalized wave function. Note that $\left\langle r^{2}\right\rangle_{n l j}$ does not depend on 
the occupancy $v_{n l j}^{2}$ or on the multiplicity $2 j+1$ of the orbital. The mean square radii of the majority of the levels are in the range of $30-50 \mathrm{fm}^{2}$ for all $\mathrm{Sn}$ isotopes, which is less than twice the total mean square radii of these isotopes. However, the mean square radii of the $3 p$ levels are much larger. They reach values of $65-90 \mathrm{fm}^{2}$ when they start to be populated at $N=90$ and of $50-55 \mathrm{fm}^{2}$ in the heaviest isotopes. Such a huge value magnifies the contribution of a few neutrons to the neutron radius when these levels are occupied. To visualize this effect, in panel (d) of Fig. 2 we have plotted the quantity $\tau_{n l j}$, defined as

$$
\tau_{n l j}=v_{n l j}^{2}\left\langle r^{2}\right\rangle_{n l j},
$$

which describes the contribution of a single neutron to the total value of the neutron mean square radius $\left\langle r^{2}\right\rangle_{n}$. Thus we may write the neutron mean square radius as

$$
\left\langle r^{2}\right\rangle_{n}=\frac{1}{N} \sum_{n l j}(2 j+1) \tau_{n l j}
$$

where $N$ is the neutron number of the isotope.

In Fig. 2(d) we see that $\tau_{n l j}$ roughly follows the pattern of $v_{n l j}^{2}$. Nevertheless, the $\tau_{n l j}$ values of the $3 p$ orbitals show a different behavior. They are magnified in comparison to the other levels. For example, for $N=98$ the $3 p_{3 / 2}$ orbital has the same impact on the neutron radius as the $2 f_{7 / 2}$ orbital, despite their occupancies being respectively 0.6 and 0.9 . Neutrons from the fully occupied $3 p$ levels have larger $\tau_{n l j}$ values than the other orbitals of the valence shell. Due to the large $\left\langle r^{2}\right\rangle_{n l j}$ value of the $3 p$ levels, neutrons from these orbitals have a very strong impact on the neutron radius.

From the knowledge of the impact of particular orbitals on the structure of the neutron surface, we can now explain the curvature of the diagrams in Fig. 1. In the first half of the shell, neutrons occupy mainly transitional (such as $2 f$ ) or high- $n$ low- $l$ (such as $3 p$ ) orbitals. They give an additional increase of the surface diffuseness of the neutron distribution. This can be seen in Fig. 3, where we compare the neutron density profile and its derivative of the magic nucleus ${ }^{132} \mathrm{Sn}$ with the midshell isotope ${ }^{154} \mathrm{Sn}$. It is clear that in ${ }^{154} \mathrm{Sn}$ the surface diffuseness is larger and that the slope of the surface fall-off is smaller. In the second half of the shell, the influence of the low- $n$-high- $l$ level $1 i_{13 / 2}$ gives the opposite effect of decreasing the surface diffuseness. In the particular example of the isotopes ${ }^{132} \mathrm{Sn}$, ${ }^{154} \mathrm{Sn}$, and ${ }^{176} \mathrm{Sn}$ we have found that $a_{n}$ takes values 0.526 , 0.815 , and $0.720 \mathrm{fm}$, respectively. Thus, the neutron density profile of ${ }^{176} \mathrm{Sn}$, also plotted in Fig. 3, has a larger slope of the density distribution at the surface than in ${ }^{154} \mathrm{Sn}$.

At this stage, let us summarize the above observations for the sake of clarity. The comparison of Figs. 1(b) and 2(d) unravels a correlation between the changes in the isotopic shift of the NST and the sp spectrum of the orbitals in the considered shell. When high- $n-$ low- $l$ orbitals are populated (e.g., $3 p_{3 / 2}$ between $N=90$ and 96), a rapid increase of the surface contribution $\Delta r_{n p}^{\text {surf }}$ to the NST of a nucleus can be noticed. It is manifested by an additional increase of the NST. Conversely, when levels with low- $n$-high- $l$ quantum numbers are being occupied (e.g., $1 i_{13 / 2}$ above $N=110$ ), the bulk contribution $\Delta r_{n p}^{\text {bulk }}$ increases while $\Delta r_{n p}^{\text {surf }}$ decreases. Population of orbitals with intermediate principal quantum number and angular

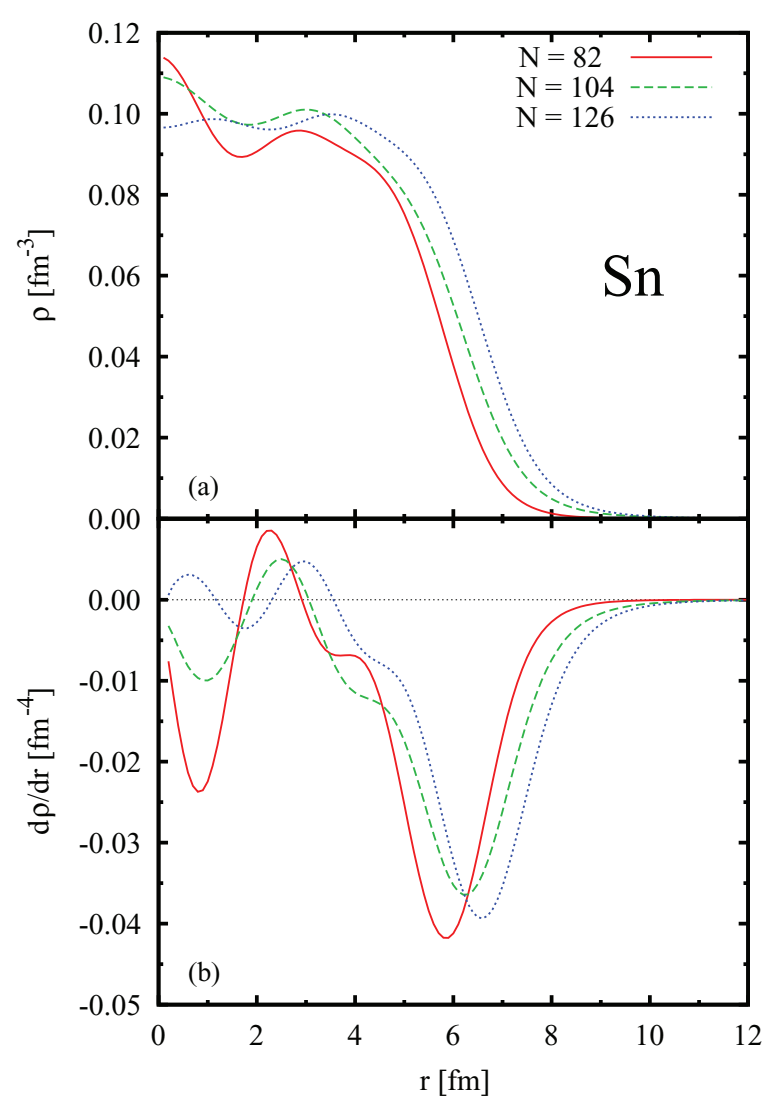

FIG. 3. (Color online) Neutron density profiles of $\mathrm{Sn}$ isotopes with $N=82, N=104$, and $N=126$ (a) and their derivatives (b).

momentum (such as the $2 f_{7 / 2}$ orbital below $N=90$ ) supports both the contributions to the NST. When several types of levels are filled simultaneously $(96 \leqslant N \leqslant 110)$ we see a combination of both effects.

\section{B. Single-particle neutron density distributions}

In order to find the source of the correlation described in the previous subsection, we have to investigate the spatial distribution of the neutrons from each particular orbital. In Fig. 4 we analyze the neutron distribution of ${ }^{176} \mathrm{Sn}$, i.e., the heaviest isotope in the considered shell with all occupied orbitals. We compare the total neutron density distribution, plotted with solid lines, with the density distributions of neutrons from each level of the valence shell (dashed lines) defined as

$$
\rho_{n l j}(r)=(2 j+1) v_{n l j}^{2} \varphi_{n l j}^{2}(r) .
$$

Indeed, the sp mean square radii defined in Eq. (6) can be expressed for fully occupied orbitals $\left(v_{n l j}^{2}=1\right)$ through $\rho_{n l j}(r)$ as

$$
\left\langle r^{2}\right\rangle_{n l j}=\frac{4 \pi}{2 j+1} \int_{0}^{\infty} d r r^{4} \rho_{n l j}(r) .
$$

The difference between the total density $\rho(r)$ and the density of each orbital $\rho_{n l j}(r)$ is also plotted in Fig. 4 with the dotted line. In this way we find how neutrons from each level of the last shell modify the total neutron density profile and how they affect the neutron surface. The behavior of the sp 


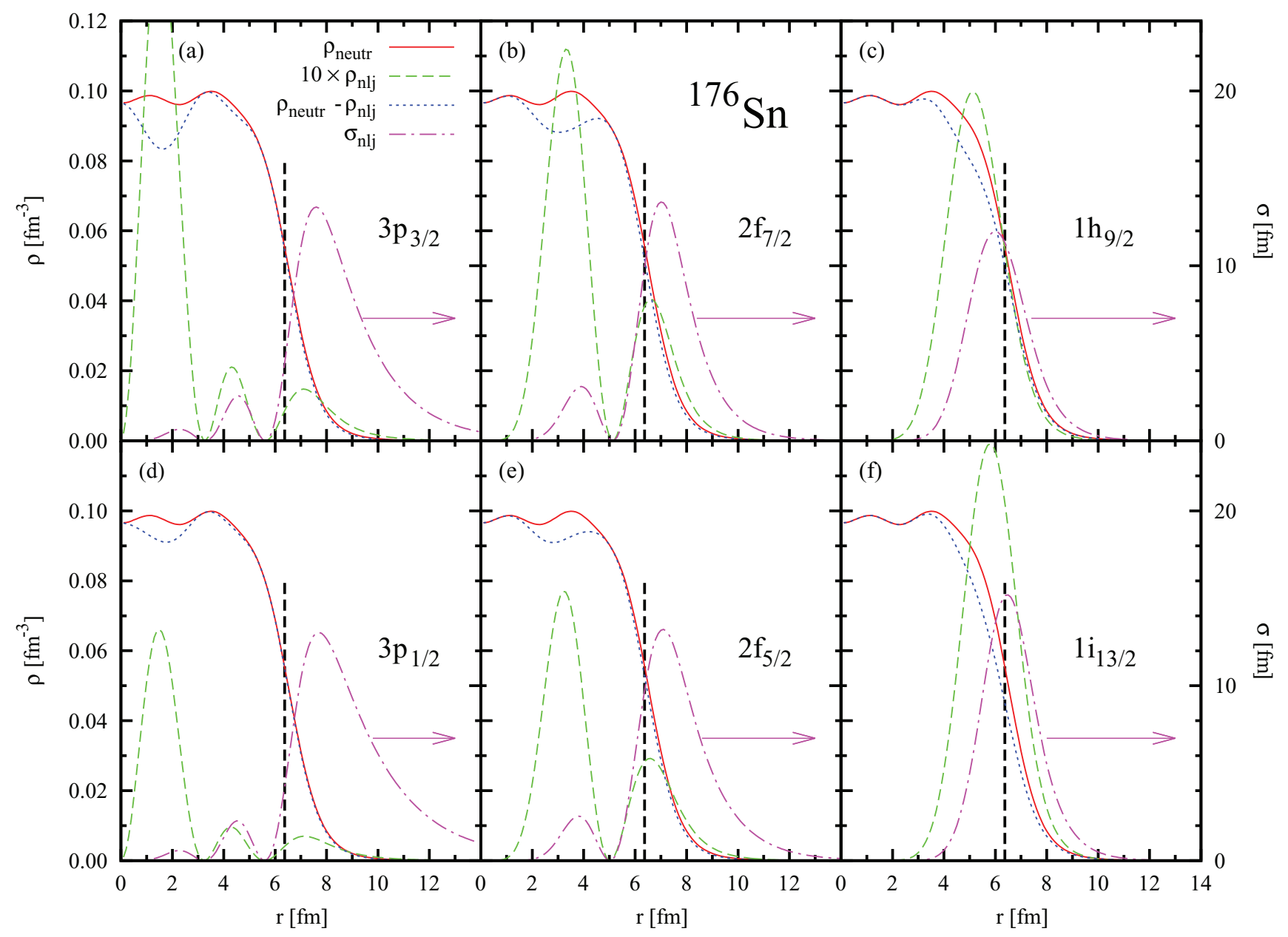

FIG. 4. (Color online) Neutron density profile (red solid line) of ${ }^{176} \mathrm{Sn}$ compared to the single-particle density $\rho_{n l j}(r)$ of an individual orbital defined in Eq. (9) (multiplied by 10, green dashed line) and their difference (blue dotted line) for the orbitals (a) $3 p_{3 / 2}$, (b) $2 f_{7 / 2}$, (c) $1 h_{9 / 2}$, (d) $3 p_{1 / 2}$, (e) $2 f_{5 / 2}$, and (f) $1 i_{13 / 2}$ as a function of the distance from the center of the nucleus. The function $\sigma_{n l j}(r)$ defined in Eq. (12) (magenta dot-dashed line) is presented on the right vertical scale. The vertical dashed line indicates the position of the half-density radius $C_{n}=6.367 \mathrm{fm}$.

density distribution of each orbital is consistent with the basic quantum mechanical properties of the nuclear orbitals.

The main contribution of the $3 p$ levels to the total density (left panels of Fig. 4) is due to the innermost bump of their $\mathrm{sp}$ densities which is located in the interior of the nucleus at distances below $3 \mathrm{fm}$ from the center. The two outer bumps of the density of the $3 p$ orbitals contribute much less to the total neutron density. In the central panels of Fig. 4 we can see that the inner bump of the $2 f$ orbitals is peaked at $r \approx 3 \mathrm{fm}$. It also mainly contributes to the bulk region of the total density. The outer bump of the $2 f$ orbitals, located at the surface, is much smaller. In contrast, neutrons from $n=1$ orbitals (right panels of Fig. 4) practically do not contribute to the total density at distances below $2 \mathrm{fm}$ from the center. The single peak of their density distribution is localized in the surface region with the maximum at $r=5-6 \mathrm{fm}$. It can be seen that the orbitals $1 h_{9 / 2}$ and $1 i_{13 / 2}$ modify the density distribution by shifting the whole surface outside and enlarging the half-density radius.

The analysis of just the density profile may not be enough when mean square (ms) or rms radii are treated as a measure of the nuclear size. In the integral defining the mean square radii of spherical nuclei, the nuclear density is weighted by the fourth power of the distance from the center:

$$
\left\langle r^{2}\right\rangle=\frac{4 \pi}{N} \int_{0}^{\infty} d r r^{4} \rho(r) .
$$

The subintegral function in this expression is peaked at the nuclear surface. Therefore $\left\langle r^{2}\right\rangle$ is much more sensitive to the shape of density profile at the surface than to the nuclear bulk density. To check the contribution of the neutrons in each sp level to the mean square radii we have to examine the sp densities multiplied by $r^{4}$. Therefore, in Fig. 4 (vertical scale on the right) we have also plotted the function $\sigma_{n l j}(r)$ (dash-dotted line) defined as

$$
\sigma_{n l j}(r)=4 \pi r^{4} v_{n l j}^{2} \varphi_{n l j}^{2}(r) .
$$

This function is the contribution of a single neutron in the $n l j$ orbital to the mean square neutron radius that for a spherically symmetric nucleus reads

$$
\left\langle r^{2}\right\rangle_{n}=\frac{1}{N} \sum_{n l j}(2 j+1) \int_{0}^{\infty} d r \sigma_{n l j}(r) .
$$


Notice that the function $\sigma_{n l j}(r)$ is related to the $\tau_{n l j}$ number, introduced before in Eq. (7), by

$$
\tau_{n l j}=\int_{0}^{\infty} d r \sigma_{n l j}(r)
$$

As expected, the function $\sigma_{n l j}(r)$ for all neutron orbitals is concentrated at the surface and vanishes in the nuclear interior. The inner bumps of $\sigma_{n l j}(r)$ in the $3 p$ and $2 f$ orbitals, unlike $\rho_{n l j}(r)$, are strongly damped in comparison with the other bumps. Some important differences can be observed in the spatial distribution of $\sigma_{n l j}(r)$ between the various types of orbitals. In the $3 p$ levels, the outermost bump of $\sigma_{n l j}(r)$ is peaked in the tail of the neutron density distribution, around $1.5 \mathrm{fm}$ from the half-density radius $C_{n}$ (indicated in Fig. 4 by the black dashed vertical line). Almost the whole contribution of $\sigma_{n l j}(r)$ to $\left\langle r^{2}\right\rangle_{n l j}$ in the $3 p$ levels comes from the region outside $C_{n}$. The values of $\sigma_{n l j}(r)$ are significant even at large distances beyond the nuclear surface where the neutron density is negligible. Thus, the two $3 p$ levels enlarge the neutron rms radius mainly by modifying the surface diffuseness.

In the transitional $2 f$ levels the maximum of $\sigma_{n l j}(r)$ is located less than $1 \mathrm{fm}$ outside $C_{n}$. Both the surface region and the tail of the density contribute to the function $\sigma_{n l j}(r)$. The influence of the $2 f$ orbitals on the neutron skin is more ambiguous than in the case of the $3 p$ orbitals because they simultaneously contribute to the surface part and to the bulk part of the NST.

The function $\sigma_{n l j}(r)$ of the low- $n$-high- $l$ neutrons $\left(1 h_{9 / 2}\right.$ and $1 i_{13 / 2}$ ) has a single bump with a maximum in the vicinity of the half-density radius $C_{n}$. It is distributed rather symmetrically around $C_{n}$ and does not extend to large distances. Neutrons from these orbitals almost do not modify the surface diffuseness $a_{n}$, but increase the half-density radius $C_{n}$. As a consequence, the surface contribution $\Delta r_{n p}^{\text {surf }}$ to the neutron skin diminishes and the bulk part $\Delta r_{n p}^{\text {bulk increases }}$ substantially.

In Fig. 5 we have plotted for ${ }^{176} \mathrm{Sn}$ the same functions as in Fig. 4 but for all neutrons from the whole valence shell.

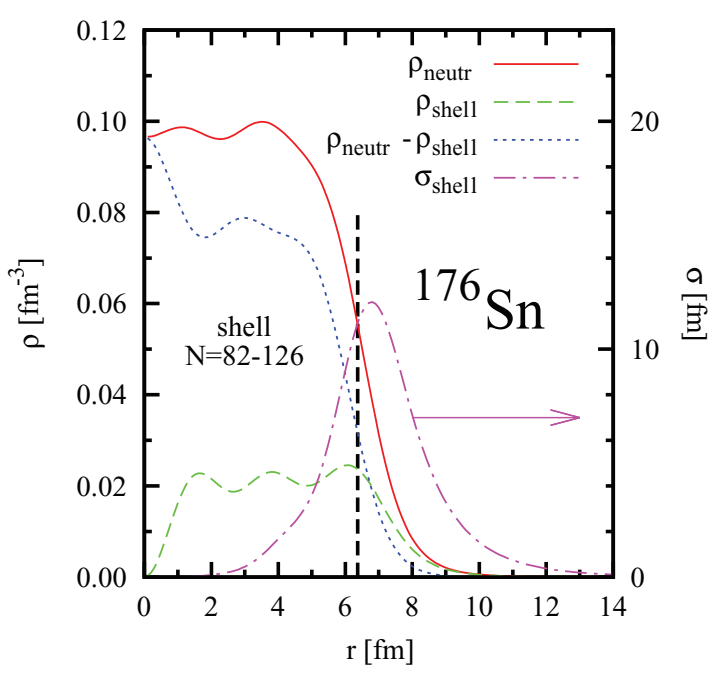

FIG. 5. (Color online) The same as in Fig. 4, but for the whole valence major shell from $N=82$ to $N=126$.
The net total contribution of the neutrons from the last shell, denoted by $\rho_{\text {shell }}(r)$, is displayed by the dashed line in Fig. 5 . It is distributed rather evenly throughout the nuclear interior. Due to the lack of $s$ orbitals in the considered shell, it vanishes in the center of the nucleus. The total density distribution in the surface region is determined mostly by the neutrons from the last shell. The contribution of $\sigma_{\text {shell }}(r)$ to the neutron mean square radius averages the contributions of all the orbitals of the valence shell. It is peaked around the half-density radius and it has quite a large width, extending to the area outside the nuclear surface. Therefore, the shell as a whole contributes to both the bulk and surface parts of the neutron skin.

\section{Structure of the tail of the neutron density distribution}

In the previous subsection, we have found that the tail of the density beyond the mean position of the nuclear surface gives a non-negligible contribution to the neutron mean square radii and is significant for the neutron skin formation. Now we will discuss the structure of the tail of the neutron densities along the valence shell of the Sn isotopic chain. In Fig. 6 we have plotted with the thick solid line the ratio of the density of the nucleons from the whole $N=82-126$ shell to the total neutron density $\rho_{\text {shell }}(r) / \rho_{\text {neutr }}(r)$ for the few selected $\mathrm{Sn}$ isotopes along the considered shell. In all these nuclei, $\rho_{\text {shell }}(r) / \rho_{\text {neutr }}(r)$ grows from $r=5 \mathrm{fm}$ and reaches $90 \%$ at $r=10-12 \mathrm{fm}$. Hence, the valence nucleons play a dominant role in the structure of the tail of the density distribution.

In Fig. 6 the ratio $\rho_{n l j}(r) / \rho_{\text {neutr }}(r)$ of the orbital density, defined in Eq. (9), to the total neutron density is also plotted [74]. The huge influence of the single neutrons on the tail of the nuclear density distribution can be noticed. In panel (a) of Fig. 6, the case of ${ }^{136} \mathrm{Sn}$ is shown, where only four neutrons mainly from the $2 f_{7 / 2}$ orbital are present in the last shell. The ratio to the total density reaches around $60 \%$ at $r=10 \mathrm{fm}$. The contribution of the $2 f_{7 / 2}$ level in heavier isotopes diminishes in favor of the other orbitals, but even in the heaviest isotopes its maximum remains at the level of a $20 \%$ contribution. In all of the isotopes, neutrons from only a few orbitals determine the total density of the nuclear tail. Even as close to the bulk as at half-density radius, selected orbitals share $10 \%-20 \%$ of the total density, e.g. $1 i_{13 / 2}$ in ${ }^{176} \mathrm{Sn}$. Despite the fact that they accomodate only six neutrons, the $3 p_{3 / 2}$ and $3 p_{1 / 2}$ levels seem to be dominant in the tail at large distances, where these two levels together account for even $80 \%$ of the neutron density.

In the analysis of the sp contribution to the tail of the neutron density, we cannot forget about the rapid, exponential decrease of the neutron density at large distances outside the nuclear surface. It is depicted in Fig. 7 where the neutron density of the nucleus ${ }^{176} \mathrm{Sn}$ is plotted in logarithmic scale. It can be noticed that beyond $r=10 \mathrm{fm}$ the neutron density of ${ }^{176} \mathrm{Sn}$ decreases below $10^{-3} \mathrm{fm}^{-3}$, which is $1 \%$ of the bulk density. At these distances only neutrons from the valence shell $N=82-126$ contribute to the total neutron density, which confirms the conclusions deduced from Fig. 6(f). When we look into the individual densities of the sp orbitals, also displayed in Fig. 7, we notice that the type of orbital determines the slope of the logarithmic decrease of the sp neutron density. The fall-off of the $3 p$ levels is slower than for the $2 f$ levels, and the largest 


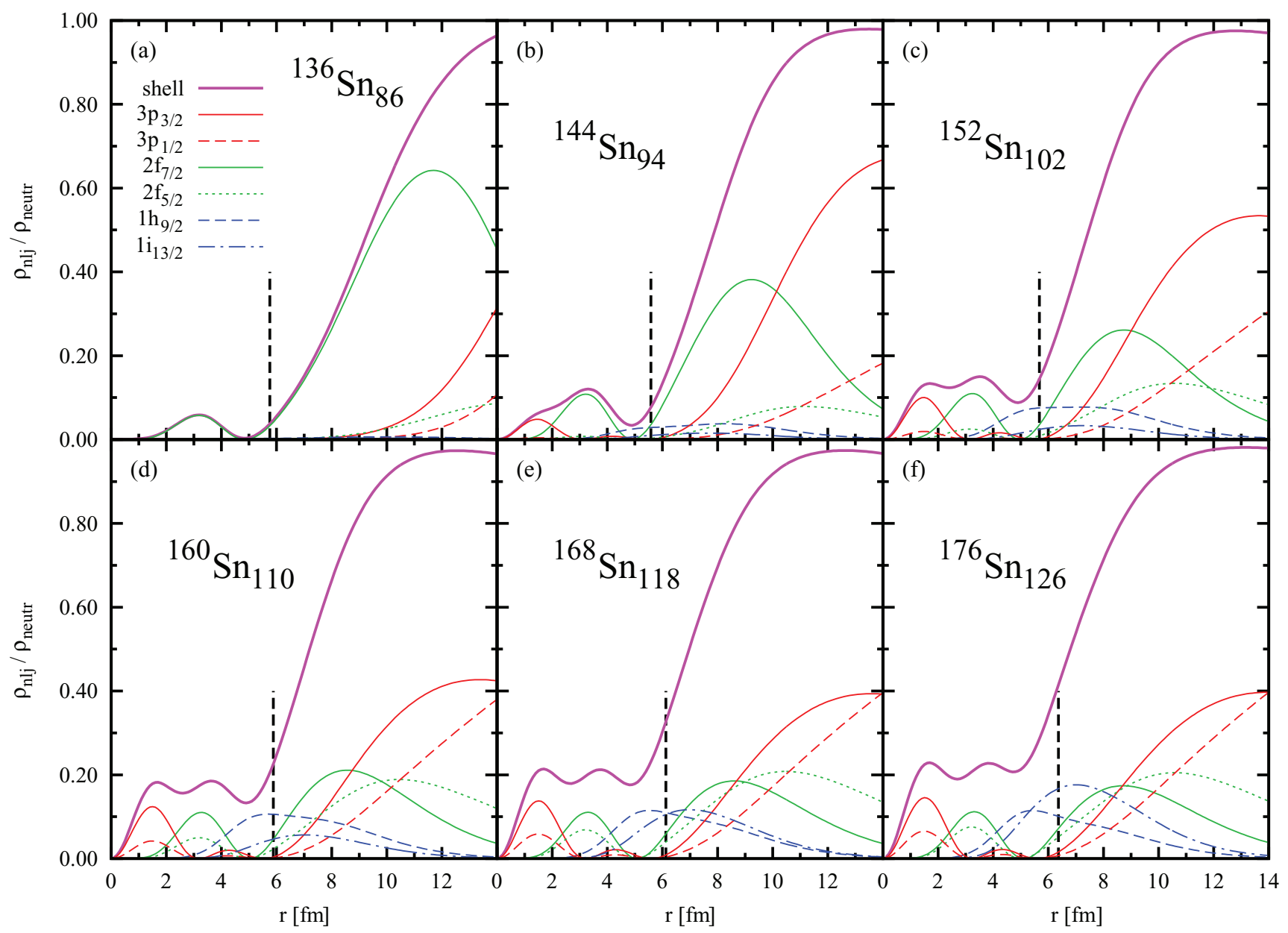

FIG. 6. (Color online) Ratio of the whole shell density (magenta thick solid line) and of the single-particle level density to the total neutron density as a function of the distance from the center of the nucleus in Sn isotopes: (a) ${ }^{136} \mathrm{Sn}$, (b) ${ }^{144} \mathrm{Sn}$, (c) ${ }^{152} \mathrm{Sn}$, (d) ${ }^{160} \mathrm{Sn}$, (e) ${ }^{168} \mathrm{Sn}$, and (f) ${ }^{176} \mathrm{Sn}$. The ratios for the $3 p_{3 / 2}$ and $3 p_{1 / 2}$ orbitals are marked by red lines, the ratios for the $2 f_{7 / 2}$ and $2 f_{5 / 2}$ orbitals by green lines, and the ratios for the $1 h_{9 / 2}$ and $1 i_{13 / 2}$ orbitals by blue lines. The vertical dashed line indicates the position of the half-density radius $C_{n}$ in each of the considered nuclei.

slope is found for the $1 h_{9 / 2}$ and $1 i_{13 / 2}$ levels. Thus, the $3 p$ levels participate much more than the low- $n$-high- $l$ levels to the total neutron density at very large distances from the center of the nucleus, as expected from their high mean square radii [cf. $\left\langle r^{2}\right\rangle_{n l j}$ in panel (c) of Fig. 4 and $\sigma_{n l j}(r)$ in Fig. 5]. At distances larger than about $4 \mathrm{fm}$ from the half-density radius, the $3 p$ levels determine the slope of the fall-off of the neutron density. We have to remember that in this region the $3 p$ orbitals still contribute to the neutron rms radii as the densities are weighted by $r^{4}$ in the calculation of this observable [cf. Eq. (11) and Figs. 4 and 5]. The shape of the tail of the nuclear density is also very important in the analysis of the experiments performed in exotic atoms such as antiprotonic or pionic atoms. The heavy, negatively charged particle annihilates at distances around 2 or $3 \mathrm{fm}$ from the nuclear surface $[10,69,70]$ and it is highly sensitive to the differences between the neutron and proton density distributions in this region.

In this section we have studied the correlation between the quantum numbers of the valence neutrons and the neutron skin of $\mathrm{Sn}$ isotopes. Neutron orbitals with low principal quantum number $n$ and high angular momentum $l$ are localized at the nuclear surface. They induce a shift of the neutron half-density radius and increase the bulk contribution to the NST. Neutron levels with high principal quantum number $n$ and low angular momentum $l$ can be found in the same shell. The outermost bump of their density distribution is localized in the tail of the neutron density profile. It brings about a large contribution to the mean square neutron radii and determines the slope of the logarithmic fall-off of the neutron density outside the surface region. The high- $n-$ low- $l$ orbitals are responsible for increasing the surface contribution to the NST.

\section{NEUTRON SKIN IN THE OTHER ISOTOPIC CHAINS}

With the experience gained in the previous section, we analyze on the same footing the other isotopic chains with magic proton number, namely $\mathrm{Ca}, \mathrm{Ni}, \mathrm{Zr}$, light $\mathrm{Sn}$ isotopes, and $\mathrm{Pb}$, which are representative of different mass regions.

\section{A. Ca and $\mathrm{Ni}$ isotopes with $20 \leqslant N \leqslant \mathbf{5 0}$}

We study $\mathrm{Ca}$ and $\mathrm{Ni}$ isotopes in the range covering the two major neutron shells $N=20-28$ and $N=28-50$. The 


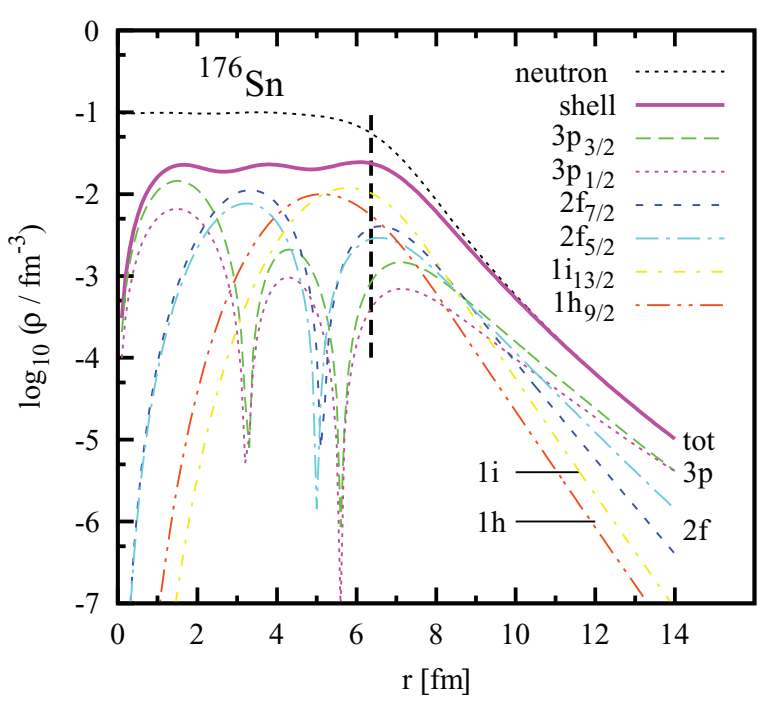

FIG. 7. (Color online) Neutron density of ${ }^{176} \mathrm{Sn}$ as a function of the distance from the center of the nucleus (black dotted line) in logarithmic scale compared to the contribution from the whole $N=$ 82-126 shell (magenta solid line) and to the individual contributions from the single-particle orbitals. The densities of the orbitals are marked by red lines for the $3 p_{3 / 2}$ and $3 p_{1 / 2}$ levels, by green lines for the $2 f_{7 / 2}$ and $2 f_{5 / 2}$ levels, and by blue lines for the $1 h_{9 / 2}$ and $1 i_{13 / 2}$ levels. The vertical dashed line indicates the position of the half-density radius $C_{n}=6.367 \mathrm{fm}$ of the nucleus.

neutron drip line of the $\mathrm{Ca}$ isotopes is reached at $N=48$ close to the upper limit of the $N=28-50$ shell, whereas it is

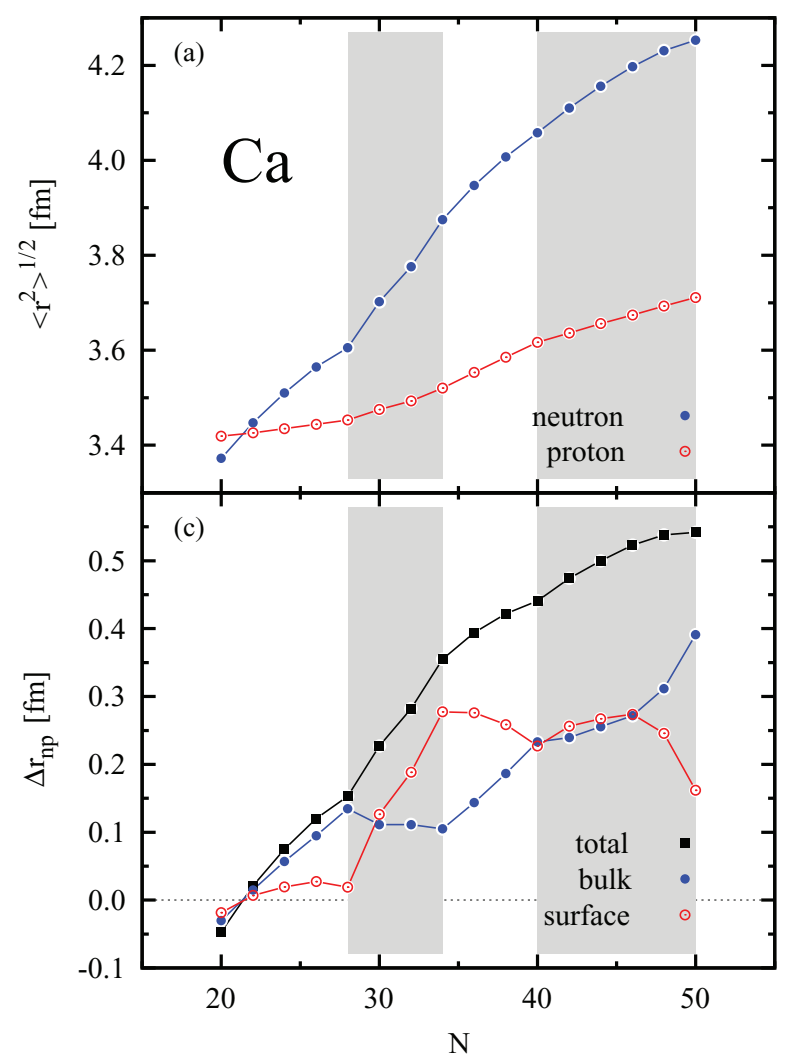

shifted to $N=60$ for Ni. In Figs. 8(a) and 8(b) we can see, similarly to the case of the $\mathrm{Sn}$ isotopes, that the proton rms radius of the $\mathrm{Ca}$ and $\mathrm{Ni}$ isotopic chains grows rather smoothly, whereas the neutron rms radius increases with a variable slope. The additional eight protons in Ni make the proton rms radii of this element larger than in $\mathrm{Ca}$ by $\sim 0.25 \mathrm{fm}$. However, the neutron rms radii change very little from $\mathrm{Ca}$ to $\mathrm{Ni}$. As a consequence, the NST in the Ni isotopes is around $0.25 \mathrm{fm}$ smaller than in $\mathrm{Ca}$ for the same number of neutrons. Indeed, in the neutron-deficient isotopes of $\mathrm{Ni}$ from $N=20$ till $N=28$, the NST [as well as its bulk and surface parts, which are plotted in Figs. 8(c) and 8(d)] is negative. The total change in absolute value of the NST from $N=20$ to $N=50$ is similar in both the $\mathrm{Ni}$ and $\mathrm{Ca}$ isotopic chains; that is, around $0.55 \mathrm{fm}$ for $\mathrm{Ni}$ and $0.59 \mathrm{fm}$ for $\mathrm{Ca}$.

Looking at the bulk $\Delta r_{n p}^{\text {bulk }}$ and surface $\Delta r_{n p}^{\text {surf }}$ contributions to the NST in Ca and Ni, one sees from Fig. 8 that the largest part of the change of the NST from Ca to Ni comes from the change of $\Delta r_{n p}^{\text {bulk }}$ between these two elements, whereas $\Delta r_{n p}^{\text {surf }}$ changes less from one element to the other element. In fact, in the case of the magic numbers $N=20, N=28$, and $N=50$ it is seen that the value of $\Delta r_{n p}^{\text {surf }}$ is almost equal in $\mathrm{Ca}$ and $\mathrm{Ni}$. Thus, the decrease of the NST from Ca to Ni in these nuclei with magic neutron number is produced almost entirely by the decrease of the bulk contribution $\Delta r_{n p}^{\text {bulk }}$.

Four intervals of the neutron number are clearly distinguished in the bulk and surface contributions to the NST in Figs. 8(c) and 8(d). In the first interval, corresponding to the $N=20-28$ shell, the bulk contribution to the NST grows almost linearly and the surface contribution remains practically

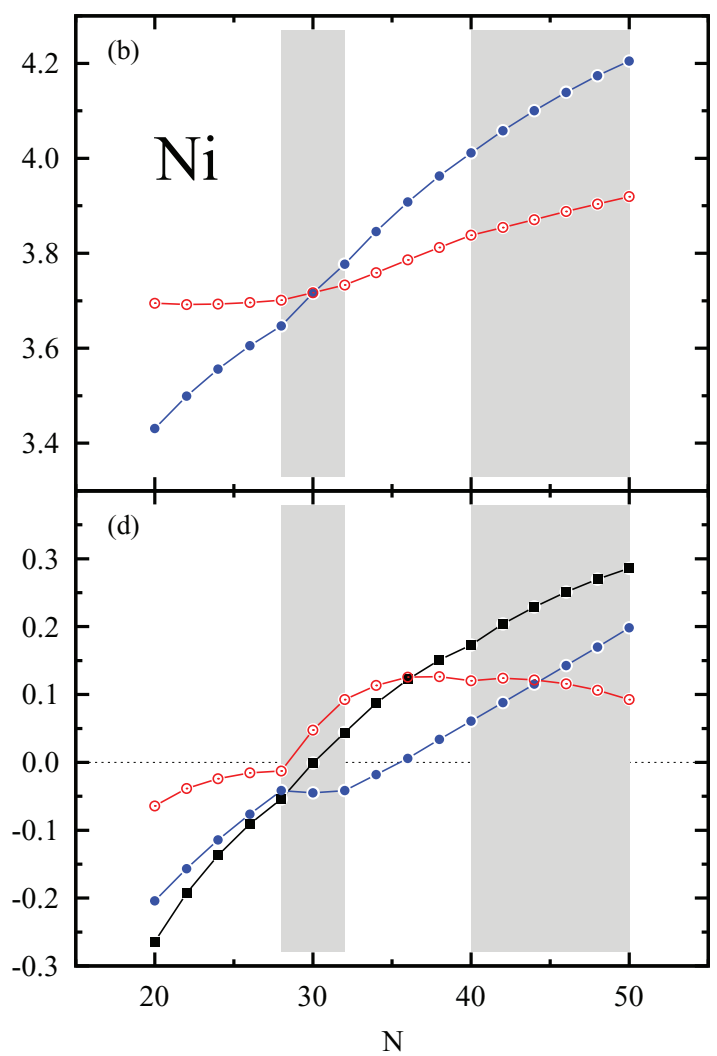

FIG. 8. (Color online) The same as in Fig. 1 but for $\mathrm{Ca}$ and Ni isotopes with $20 \leqslant N \leqslant 50$. 


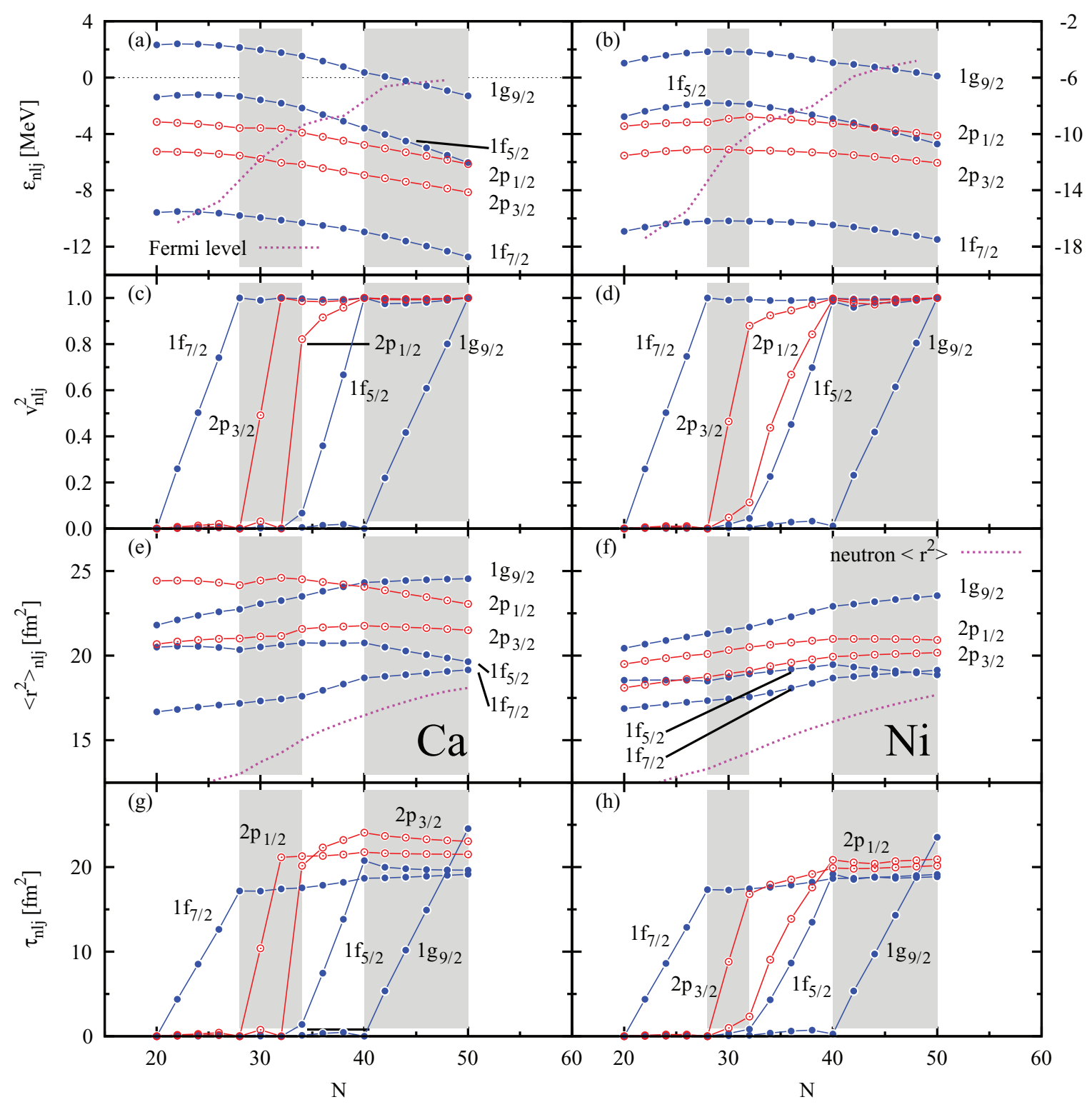

FIG. 9. (Color online) The same as in Fig. 2 but for $\mathrm{Ca}$ and $\mathrm{Ni}$ isotopes with $20 \leqslant N \leqslant 50$. Note that the vertical scale of panel (b) is on the right axis.

constant. The low- $n$-high- $l$ level $1 f_{7 / 2}$ is populated within this region, as it can be seen from panels (a)-(d) of Fig. 9 which display the position and occupation of the levels close to the Fermi energy for the $\mathrm{Ca}$ and $\mathrm{Ni}$ isotopes. The second interval of the neutron number ranges from $N=28$ to $N=34$ in $\mathrm{Ca}$ and to $N=32$ in Ni. In these nuclei the surface contribution to the NST grows rapidly while the bulk part slightly decreases. The $2 p_{3 / 2}$ level is being filled mostly in this interval as well as the $2 p_{1 / 2}$ level in Ca [cf. Figs. 9(a)-9(d)].

The third interval of Fig. 8 covers the isotopes up to $N=40$ where the bulk part of the NST increases and the surface part slightly decreases $(\mathrm{Ca})$ or remains almost constant $(\mathrm{Ni})$. Within this region, in Ca mainly the $1 f_{5 / 2}$ level is being occupied. The $2 p_{1 / 2}$ level lies closer to the $1 f_{5 / 2}$ level in $\mathrm{Ni}$ than in $\mathrm{Ca}$, and consequently, these two orbitals in $\mathrm{Ni}$ are populated almost simultaneously between $N=32$ and $N=40$ [cf. Figs. 9(a)-9(d)]. Thus, the third interval in neutron number of the $\mathrm{Ni}$ isotopes starts already at $N=32$, rather than at $N=34$ as in $\mathrm{Ca}$. In this third interval of $\mathrm{Ni}$ the influence of the $2 p_{1 / 2}$ orbital on the NST of Ni is overpowered by neutrons from the $1 f_{5 / 2}$ orbital; one finds a very modest rise of the surface part $\Delta r_{n p}^{\text {surf }}$ of the NST while the bulk part $\Delta r_{n p}^{\text {bulk }}$ grows linearly [see Fig. 8(d)].

The fourth interval of Fig. 8 ranges between $N=40$ and $N=50$, where occupation of the $1 g_{9 / 2}$ level takes place. In Ca between $N=40$ and $N=46$ both the bulk and surface contributions to the NST take a similar value with a slight growing trend with $N$. For larger number of neutrons in the $\mathrm{Ca}$ isotopes, $\Delta r_{n p}^{\text {bulk }}$ increases faster again whereas $\Delta r_{n p}^{\text {surf }}$ decreases till reaching $N=50$. In $\mathrm{Ni}$, from the semimagic number $N=40$ till $N=50$ a clear linear increase of $\Delta r_{n p}^{\text {bulk }}$ is accompanied by a slow decrease of $\Delta r_{n p}^{\text {surf }}$. This behavior 


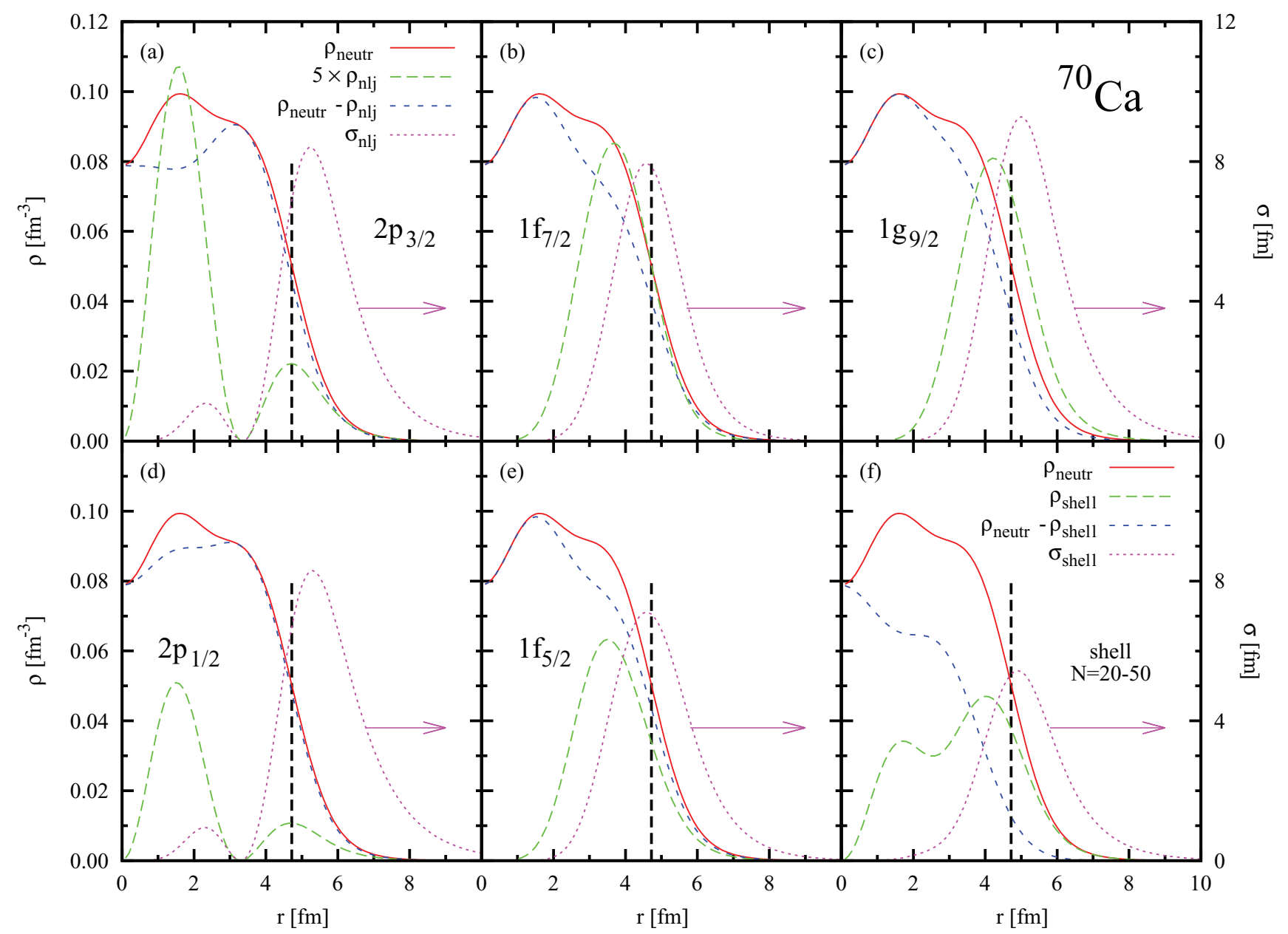

FIG. 10. (Color online) The same as in Figs. 4 and 5 but for ${ }^{70} \mathrm{Ca}$ in panels (a)-(f) and for the $20 \leqslant N \leqslant 50$ shell of Ca in panel (g).

is in agreement with the general trend for the low- $n$-high- $l$ orbitals, as in Ni only the $1 g_{9 / 2}$ level is populated.

The source of the changes in the behavior of the NST becomes clear in Fig. 9 where the sp properties of the valence orbitals are displayed. We can discuss them relying on the analysis performed in the previous section for $\mathrm{Sn}$ isotopes. In Fig. 9 it can be seen that the neutron orbitals in the vicinity of the Fermi level are rather well separated in energy. Hence, we expect quite clear differences in $\Delta r_{n p}^{\text {bulk }}$ and $\Delta r_{n p}^{\text {surf }}$ between neutron number intervals.

The $2 p$ orbitals of $\mathrm{Ca}$ and Ni play a similar role to the $3 p$ levels in Sn. They should contribute mainly to the surface of the neutron distribution. Indeed, a sudden increase of $\Delta r_{n p}^{\text {surf }}$ is observed in the second interval of Fig. 8. It can be noticed from Fig. 9 that in $\mathrm{Ca}$ and $\mathrm{Ni}$ the mean square radii of the $2 p$ levels are similar to those of the neighboring levels, unlike the case of the $3 p$ orbitals in the $\mathrm{Sn}$ isotopes. The total neutron density and the sp densities $\rho_{n l j}(r)$ of the individual neutron orbitals defined in Eq. (9), as well as the function $\sigma_{n l j}(r)$ defined in Eq. (12), are plotted in Fig. 10 for the ${ }^{70}$ Ca nucleus. Though the peak of the $\sigma_{n l j}(r)$ function of the $2 p$ levels of Ca lies closer to the half-density radius than in the case of the $3 p$ levels of $\mathrm{Sn}$ (cf. Fig. 4), the $2 p$ levels still give an important contribution to the tail of the neutron density.
The low- $n$-high- $l$ levels $1 f_{7 / 2}, 1 f_{5 / 2}$, and $1 g_{9 / 2}$ are expected to contribute to the NST by increasing the bulk part. In fact, in the first, third, and fourth intervals of Fig. 8 we see a clear increase of $\Delta r_{n p}^{\text {bulk }}$. In the Ca chain the last low- $n-$ high- $l$ orbital $1 g_{9 / 2}$ is bound only for $N>42$ with energy close to zero, as seen in panel (a) of Fig. 9. The continuum affects the behavior of the $\mathrm{Ca} 1 g_{9 / 2}$ orbital at large distances from the nuclear center. This almost unbound orbital extends to a region far away from the nucleus. Consequently, the tail of the $\sigma_{n l j}(r)$ function for the $1 g_{9 / 2}$ level [see panel (c) of Fig. 10] is larger than for the $1 f$ levels (central panels of Fig. 10). Hence, a relatively large impact of the $1 g_{9 / 2}$ level in the surface contribution to the NST of the Ca isotopes beyond $N=40$ is observed in Fig. 8(c). On the other hand, in Ni isotopes the $1 g_{9 / 2}$ level is shifted down by around $5 \mathrm{MeV}$ in comparison to $\mathrm{Ca}$, owing to the larger number of protons in $\mathrm{Ni}$. Its energy is far below zero and therefore in Ni the neutron skin splitting in bulk and surface parts for this low- $n$-high- $l$ orbital is not disturbed by the influence of the continuum, unlike in $\mathrm{Ca}$.

\section{B. $\mathrm{Zr}$ and $\mathrm{Sn}$ isotopes with $\mathbf{5 0} \leqslant N \leqslant 82$}

We have considered the shell with $N=50-82$ neutrons in the $\mathrm{Zr}$ and $\mathrm{Sn}$ elements [60]. The neutron drip line of the 

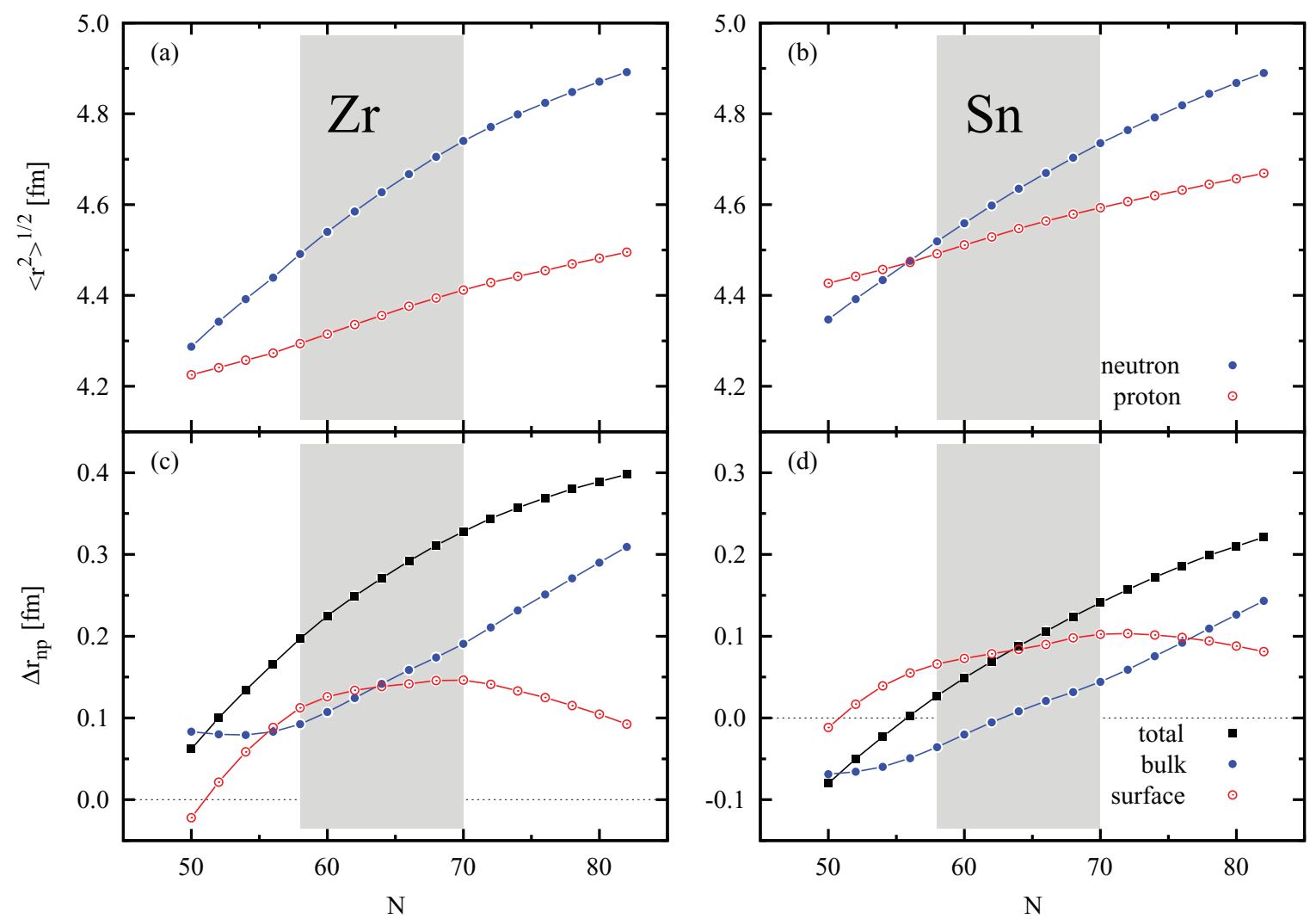

FIG. 11. (Color online) The same as in Fig. 1 but for $\mathrm{Zr}$ and $\mathrm{Sn}$ isotopes with $50 \leqslant N \leqslant 82$.

$\mathrm{Zr}$ isotopes is reached at the end of this range. In Fig. 11 we can see again the roughly linear increase of the proton rms radii with larger $N$, whereas the neutron rms radii, as well as the NST, rise with a rather curved shape. We can also see that the difference in proton number between the two elements affects mainly the proton rms radii, shifting them by a practically constant value of around $0.2 \mathrm{fm}$ along the shell. The neutron rms radii, however, remain very similar in the two elements. As a consequence, the NST decreases from Zr to $\mathrm{Sn}$ by the same amount as the proton radii increase from $\mathrm{Zr}$ to $\mathrm{Sn}$.

If we compare the values of the bulk and surface contributions to the NST in $\mathrm{Zr}$ with the values of these contributions in $\mathrm{Sn}$, it turns out that the bulk contribution $\Delta r_{n p}^{\text {bulk }}$ has decreased from $\mathrm{Zr}$ to $\mathrm{Sn}$ by a roughly constant shift of $0.15 \mathrm{fm}$, whereas the surface contribution $\Delta r_{n p}^{\text {surf }}$ remains almost the same or decreases by no more than $0.05 \mathrm{fm}$ from $\mathrm{Zr}$ to $\mathrm{Sn}$. Altogether, it implies that the change of the NST between the two elements takes place, in essence, through the modification of the sharp radius of the proton density distribution caused by the different proton number, whereas the surface diffuseness remains less affected. Actually, it is interesting to observe that in the nuclei with neutron magic number $(N=50$ and $N=82)$ the value of $\Delta r_{n p}^{\text {surf }}$ is practically identical in $\mathrm{Zr}$ and $\mathrm{Sn}$, which means that in these nuclei the change of $\Delta r_{n p}$ between $\mathrm{Zr}$ and $\mathrm{Sn}$ is exclusively due to the change of $\Delta r_{n p}^{\text {bulk }}$. These observations are in consonance with what we had found before in the study of the NST in Ca and Ni.
The bulk and the surface contributions to the NST in the $\mathrm{Zr}$ and $\mathrm{Sn}$ isotopes, unlike in the previously discussed $\mathrm{Ca}$ and $\mathrm{Ni}$ elements, change their slopes rather smoothly without any kinks in the graph [see Figs. 11(c) and 11(d)]. Nevertheless, three intervals can be distinguished. In the first interval, up to $N=58$, the surface part grows linearly. In the second interval with $N=58-70$, the surface contribution is roughly constant around its maximal value. In the third interval, beyond $N=70$, $\Delta r_{n p}^{\text {surf }}$ decreases. The bulk contribution remains practically constant up to $N=58$ and for heavier isotopes it increases linearly up to the end of the shell.

Five levels from the major shell with $N=50-82$ can be found in the energy spectrum of the $\mathrm{Zr}$ and $\mathrm{Sn}$ isotopes, as displayed in Fig. 12. There are two low- $n$-high- $l$ levels: $1 g_{7 / 2}$ and $1 h_{11 / 2}$; and three high- $n$-low- $l$ levels: $2 d_{5 / 2}, 2 d_{3 / 2}$, and $3 s_{1 / 2}$. In the first interval till $N=58$, the $2 d_{5 / 2}$ level is being filled almost without interference from the other orbitals [see panels (c) and (d) of Fig. 12]. Similarly, in the last interval beyond $N=70$ mainly the $1 h_{11 / 2}$ level is being populated. It explains the behavior shown in Figs. 11(c) and 11(d) by the bulk and surface contributions to the NST in the regions between $N=50-58$ and $N=70-82$ of the $\mathrm{Zr}$ and $\mathrm{Sn}$ isotopic chains. In the intermediate region between $N=58$ and $N=70$, the contribution of the $2 d_{5 / 2}$ level almost saturates and the $1 h_{11 / 2}$ level practically does not yet contribute to the neutron radii. The remaining $3 s_{1 / 2}, 2 d_{3 / 2}$, and $1 g_{7 / 2}$ levels play the main role in this interval. The influence of the $3 s_{1 / 2}$ and $2 d_{3 / 2}$ orbitals keeps $\Delta r_{n p}^{\text {surf }}$ from falling 


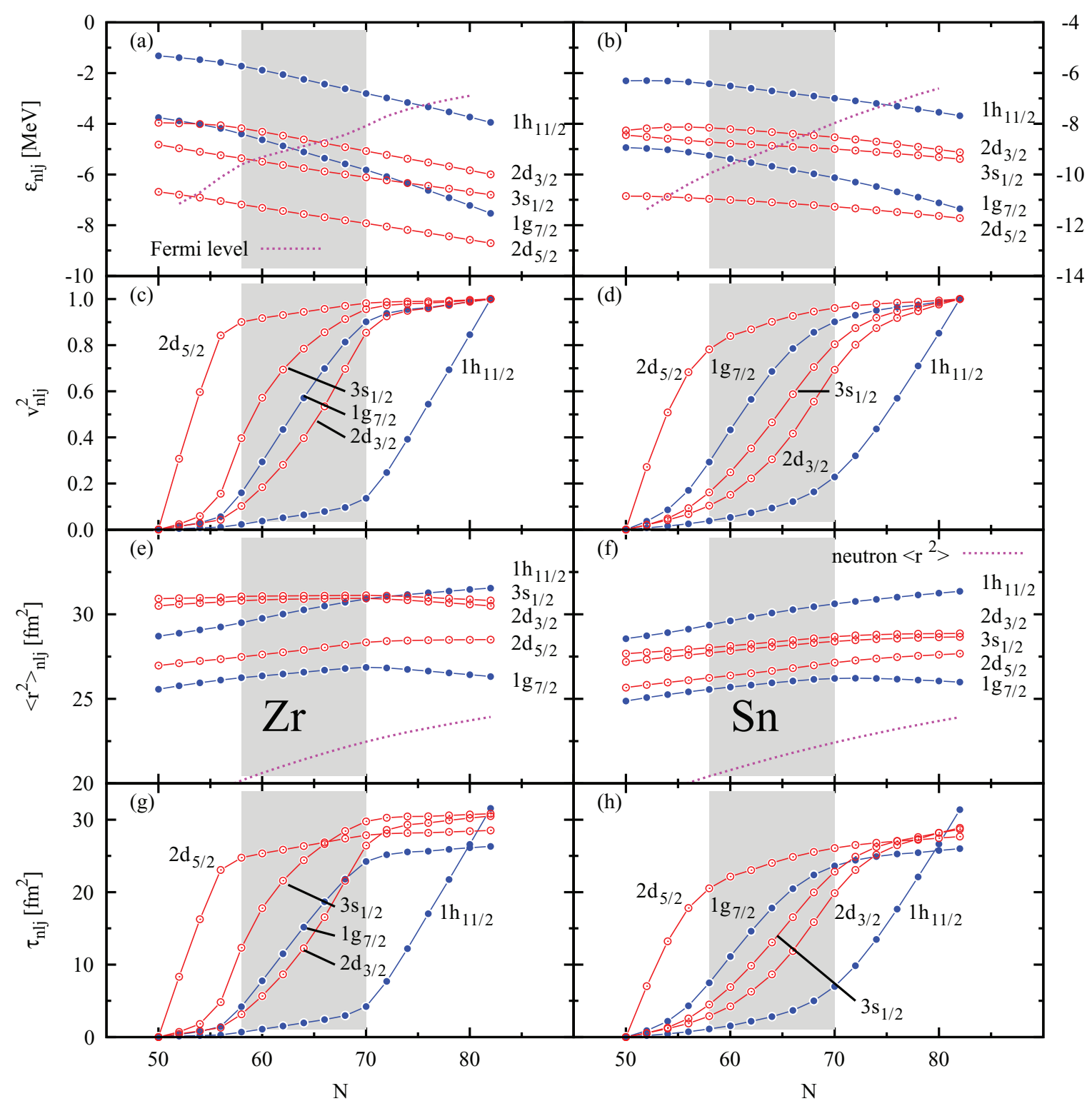

FIG. 12. (Color online) The same as in Fig. 2 but for $\mathrm{Zr}$ and $\mathrm{Sn}$ isotopes with $50 \leqslant N \leqslant 82$. Note that the vertical scale of panel (b) is on the right axis.

down, whereas the presence of the $1 g_{7 / 2}$ orbital makes $\Delta r_{n p}^{\text {bulk }}$ increase.

\section{Pb isotopes with $126 \leqslant N \leqslant 184$}

The last element considered in this article is $\mathrm{Pb}$. We concentrate on neutron-rich isotopes with $126 \leqslant N \leqslant 184$. The $\mathrm{Pb}$ neutron drip line is reached at the end of this shell. In Fig. 13 we can see an almost linear behavior of the rms radii for both protons and neutrons. Also the NST and its bulk and surface contributions are overall roughly linear with $N$. As it can be seen in Fig. 14, the large level density around the Fermi energy in these heavy nuclei causes the occupancy of all orbitals to rise along the whole shell. Hence, the influence of the sp properties onto the nuclear surface is smoothed out in this element. Nevertheless, the slight changes of the slope of the bulk and surface contributions to the NST, which can be seen in panel (b) of Fig. 13, allow one to divide the shell into four regions. In order to explain these variations we shall look into the orbitals from this valence shell, which are displayed in Fig. 14. There are seven different levels in this shell. We classify them into three groups: the low- $n-$ high- $l$ levels $1 i_{11 / 2}$ and $1 j_{15 / 2}$, the transitional levels $2 g_{9 / 2}$ and $2 g_{7 / 2}$, and the high- $n$-low- $l$ levels $3 d_{5 / 2}, 3 d_{3 / 2}$, and $4 s_{1 / 2}$. Although the occupancy of all levels changes throughout the shell, in each interval the occupancy of some orbitals increases much faster than the occupancy of the other orbitals.

In the first interval, up to $N=136$, the bulk and the surface parts of the NST increase with a similar slope. The occupancy of the transitional $2 g_{9 / 2}$ level grows very fast here. In the second region, between $N=136$ and 148, the low- $n$-high$l 1 i_{11 / 2}$ level is mostly populated. This fact is related with 


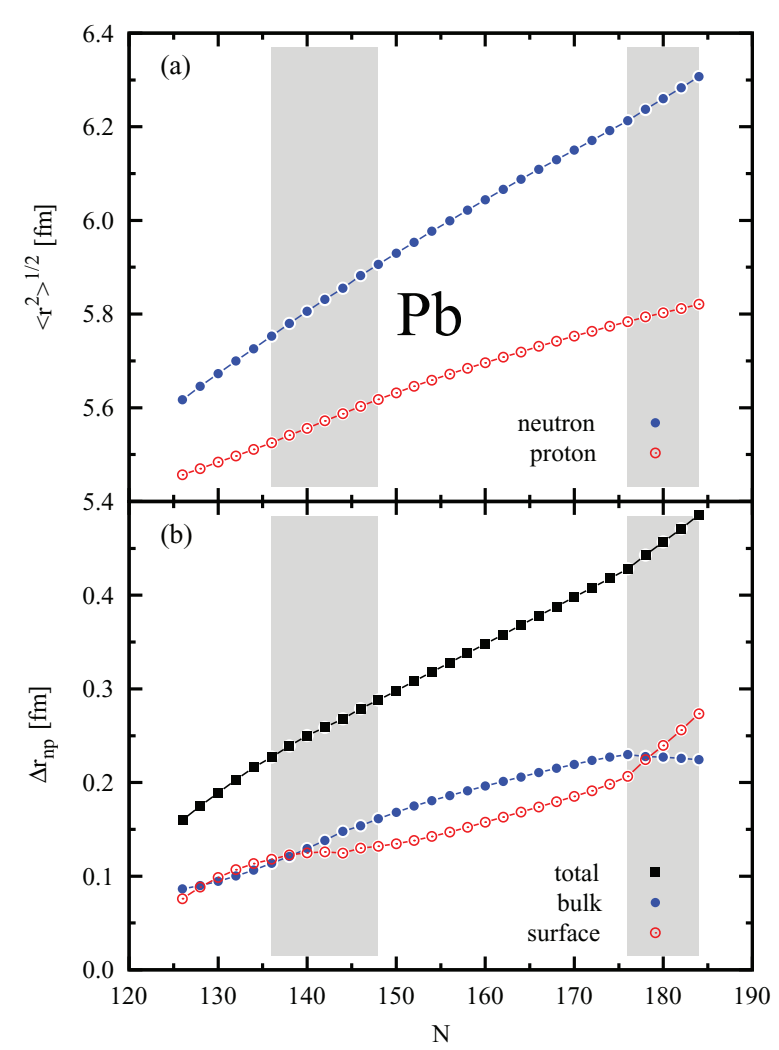

FIG. 13. (Color online) The same as in Fig. 1 but for $\mathrm{Pb}$ isotopes with $126 \leqslant N \leqslant 184$.

the observed faster increase of the bulk contribution than of the surface contribution in this second region. In the third interval, with $N=148-176$, mainly three orbitals of different type, i.e., the $1 j_{15 / 2}, 2 g_{7 / 2}$, and $3 d_{5 / 2}$ orbitals, are populated between $N=148$ and 176. Their impact on the neutron skin properties is mixed and the slopes of $\Delta r_{n p}^{\text {bulk }}$ and $\Delta r_{n p}^{\text {surf }}$ are similar again. Finally, for $N \geqslant 176$, the surface part grows faster while the bulk part slightly decreases. In this region, two levels of the high- $n-$ low- $l$ type $\left(4 s_{1 / 2}\right.$ and $\left.3 d_{3 / 2}\right)$ rapidly increase their occupancy. Their influence is magnified by their relatively large sp mean square radii $\left(\left\langle r^{2}\right\rangle_{n l j}>60 \mathrm{fm}^{2}\right)$, which can be seen in panel (c) of Fig. 14. It explains the fast increase of the surface part of the NST in this region. In spite of the small multiplicity of these levels (six neutrons only), in the closed shell nucleus with $N=184$ one finds the largest $\tau_{n l j}$ values for the $4 s_{1 / 2}$ and $3 d_{3 / 2}$ orbitals [see Fig. 14(d)].

\section{CONCLUSIONS}

We have studied the influence of the properties of the singleparticle orbitals of neutrons filling the valence shell on the nuclear surface and the neutron skin. The spherical density distributions obtained with the SLy4 mean-field interaction for the elements $\mathrm{Ca}, \mathrm{Ni}, \mathrm{Zr}, \mathrm{Sn}$, and $\mathrm{Pb}$ were examined in our investigation. Though the basic results emphasized here are general enough, the fine details may depend to some extent on the nuclear interaction.

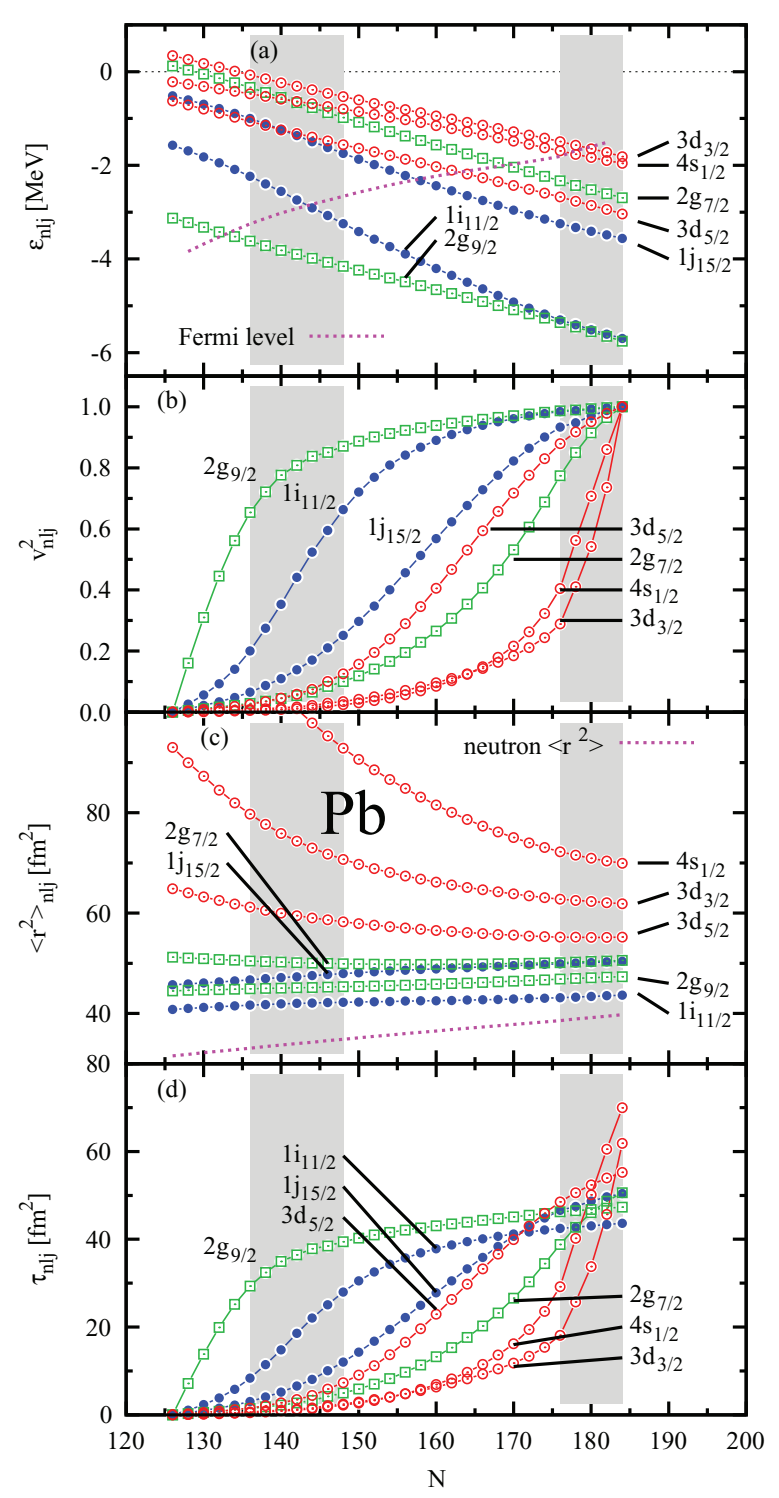

FIG. 14. (Color online) The same as in Fig. 2 but for $\mathrm{Pb}$ isotopes with $126 \leqslant N \leqslant 184$.

The single-particle mean square radii of neutron valenceshell orbitals are typically larger than the total neutron mean square radius of the nucleus. These neutron orbitals impact on the nuclear surface mainly by shifting or modifying the fall-off of the neutron density distribution. Hence, the single-particle shell structure induces changes in the behavior of the neutron skin thickness with respect to the smooth trend of the neutron skin described by models of average nuclear properties such as the droplet model.

The splitting of the neutron skin thickness into bulk and surface contributions is useful because it allows one to describe in a relatively simple way how the neutron skin is formed. When levels with low principal quantum number $n$ and large angular momentum $l$ are populated, the bulk part of the neutron skin thickness tends to grow fast. The neutrons from such orbitals are mainly localized at the surface of the neutron density. They basically shift the position of 
the neutron surface outwards without altering the density slope.

The single-particle density distribution of neutrons from orbitals with high $n$ and low $l$ has a bump beyond the neutron half-density radius of the nucleus. It enhances the diffuseness of the nuclear surface and the surface contribution to the neutron skin thickness. These levels play also a dominant role in the tail of the neutron density distribution at distances a few fm outside the surface. They govern the slope of the exponential fall-off of the neutron density in this region.

Neutron orbitals such as $3 p$ in the valence shell of Sn or $4 s$ in the valence shell of $\mathrm{Pb}$, have a very large single-particle mean square radius compared with the neighboring neutron orbitals. It magnifies the role of these levels in increasing the surface contribution to the neutron skin thickness despite the comparatively low number of neutrons that can be accommodated in them.
In this paper we have analyzed in detail the impact of the single-particle structure on the neutron skin thickness assuming spherical symmetry. However, this condition is not always met in the whole range of mass and atomic numbers considered here, as in the case of some of the $\mathrm{Zr}$ isotopes. It is known that nuclear deformations are another factor that can induce deviations of the neutron skin thickness from the average trend [55]. The study of the effects of deformation is left for a future work.

\section{ACKNOWLEDGMENTS}

This work has been partially funded by the Spanish Consolider-Ingenio 2010 Programme CPAN CSD200700042, by Grant No. FIS2011-24154 from MICINN and FEDER (Spain), and by Grant No. 2009SGR-1289 from Generalitat de Catalunya.
[1] G. Fricke, C. Bernhardt, K. Heilig, L. Schaller, L. Schellenberg, E. Shera, and C. Dejager, At. Data Nucl. Data Tables 60, 177 (1995).

[2] H. D. Vries, C. D. Jager, and C. D. Vries, At. Data Nucl. Data Tables 36, 495 (1987).

[3] I. Angeli and K. Marinova, At. Data Nucl. Data Tables 99, 69 (2013).

[4] G. W. Hoffmann et al., Phys. Rev. C 21, 1488 (1980).

[5] B. C. Clark, L. J. Kerr, and S. Hama, Phys. Rev. C 67, 054605 (2003).

[6] C. Batty, E. Friedman, and A. Gal, Nucl. Phys. A 592, 487 (1995).

[7] A. Krasznahorkay, H. Akimune, A. van den Berg, N. Blasi, S. Brandenburg, M. Csatlós, M. Fujiwara, J. Gulyás, M. Harakeh, M. Hunyadi et al., Nucl. Phys. A 731, 224 (2004).

[8] A. Trzcińska, J. Jastrzębski, P. Lubiński, F. J. Hartmann, R. Schmidt, T. von Egidy, and B. Kłos, Phys. Rev. Lett. 87, 082501 (2001).

[9] J. Jastrzȩbski, A. Trzcińska, P. Lubiński, B. Kłos, F. J. Hartmann, T. von Egidy, and S. Wycech, Int. J. Mod. Phys. E 13, 343 (2004).

[10] B. Kłos, A. Trzcińska, J. Jastrzȩbski, T. Czosnyka, M. Kisieliński, P. Lubiński, P. Napiorkowski, L. Pieńkowski, F. J. Hartmann, B. Ketzer et al., Phys. Rev. C 76, 014311 (2007).

[11] A. Klimkiewicz et al., Acta Phys. Pol. B 40, 589 (2009).

[12] J. Zenihiro, H. Sakaguchi, T. Murakami, M. Yosoi, Y. Yasuda, S. Terashima, Y. Iwao, H. Takeda, M. Itoh, H. P. Yoshida et al., Phys. Rev. C 82, 044611 (2010).

[13] D. P. Watts, for the CrystalBall@MAMI Collaboration, EPJ Web Conf. 37, 01027 (2012).

[14] C. M. Tarbert et al., arXiv:1311.0168.

[15] S. Abrahamyan, Z. Ahmed, H. Albataineh, K. Aniol, D. S. Armstrong, W. Armstrong, T. Averett, B. Babineau, A. Barbieri, V. Bellini et al. (PREX Collaboration), Phys. Rev. Lett. 108, 112502 (2012).

[16] C. Horowitz, K. Kumar, and R. Michaels, Eur. Phys. J. A 50, 48 (2014).

[17] C. Sfienti, talk given at the Workshop to Explore Physics Opportunities with Intense, Polarized Electron Beams up to $300 \mathrm{MeV}$ and the Calcium Radius Experiment (CREX) Workshop at Jefferson Lab, 2013 (unpublished).
[18] M. Centelles, X. Roca-Maza, X. Viñas, and M. Warda, Phys. Rev. Lett. 102, 122502 (2009).

[19] M. Warda, X. Viñas, X. Roca-Maza, and M. Centelles, Phys. Rev. C 80, 024316 (2009).

[20] L.-W. Chen, C. M. Ko, and B.-A. Li, Phys. Rev. Lett. 94, 032701 (2005).

[21] R. Furnstahl, Nucl. Phys. A 706, 85 (2002).

[22] M. Kortelainen, J. Erler, W. Nazarewicz, N. Birge, Y. Gao, and E. Olsen, Phys. Rev. C 88, 031305(R) (2013).

[23] Z. Zhang and L.-W. Chen, Phys. Lett. B 726, 234 (2013).

[24] J. Piekarewicz, Eur. Phys. J. A 50, 25 (2014).

[25] G. Colò, U. Garg, and H. Sagawa, Eur. Phys. J. A 50, 26 (2014).

[26] L. Trippa, G. Colò, and E. Vigezzi, Phys. Rev. C 77, 061304 (2008).

[27] X. Roca-Maza et al., Phys. Rev. C 88, 024316 (2013).

[28] A. Klimkiewicz, N. Paar, P. Adrich, M. Fallot, K. Boretzky, T. Aumann, D. Cortina-Gil, U. D. Pramanik, T. W. Elze, H. Emling et al. (LAND Collaboration), Phys. Rev. C 76, 051603 (2007).

[29] X. Roca-Maza, G. Pozzi, M. Brenna, K. Mizuyama, and G. Colò, Phys. Rev. C 85, 024601 (2012).

[30] D. Vretenar, Y. F. Niu, N. Paar, and J. Meng, Phys. Rev. C 85, 044317 (2012).

[31] B. A. Brown, Phys. Rev. Lett. 85, 5296 (2000).

[32] S. Typel and B. A. Brown, Phys. Rev. C 64, 027302 (2001).

[33] A. E. L. Dieperink, Y. Dewulf, D. Van Neck, M. Waroquier, and V. Rodin, Phys. Rev. C 68, 064307 (2003).

[34] S. S. Avancini, J. R. Marinelli, D. P. Menezes, M. M. W. Moraes, and C. Providência, Phys. Rev. C 75, 055805 (2007).

[35] M. Centelles, X. Roca-Maza, X. Viñas, and M. Warda, Phys. Rev. C 82, 054314 (2010).

[36] X. Roca-Maza, M. Centelles, X. Viñas, and M. Warda, Phys. Rev. Lett. 106, 252501 (2011).

[37] X. Viñas, M. Centelles, X. Roca-Maza, and M. Warda, Eur. Phys. J. A 50, 27 (2014).

[38] M. K. Gaidarov, A. N. Antonov, P. Sarriguren, and E. M. de Guerra, Phys. Rev. C 85, 064319 (2012).

[39] T. Gaitanos, M. D. Toro, S. Typel, V. Baran, C. Fuchs, V. Greco, and H. H. Wolter, Nucl. Phys. A 732, 24 (2004). 
[40] D. V. Shetty, S. J. Yennello, and G. A. Souliotis, Phys. Rev. C 76, 024606 (2007).

[41] B.-A. Li, L.-W. Chen, and C. M. Ko, Phys. Rep. 464, 113 (2008).

[42] M. B. Tsang, Y. Zhang, P. Danielewicz, M. Famiano, Z. Li, W. G. Lynch, and A. W. Steiner, Phys. Rev. Lett. 102, 122701 (2009).

[43] D. Vretenar, P. Finelli, A. Ventura, G. A. Lalazissis, and P. Ring, Phys. Rev. C 61, 064307 (2000).

[44] C. J. Horowitz, S. J. Pollock, P. A. Souder, and R. Michaels, Phys. Rev. C 63, 025501 (2001).

[45] O. Moreno, E. M. de Guerra, P. Sarriguren, and J. M. Udías, J. Phys. G: Nucl. Part. Phys. 37, 064019 (2010).

[46] J. Liu, Z. Ren, and T. Dong, Nucl. Phys. A 888, 45 (2012); 900, 1 (2013).

[47] A. Meucci, M. Vorabbi, C. Giusti, F. D. Pacati, and P. Finelli, Phys. Rev. C 87, 054620 (2013).

[48] D. Vretenar, G. A. Lalazissis, and P. Ring, Phys. Rev. C 62, 045502 (2000).

[49] T. Sil, M. Centelles, X. Viñas, and J. Piekarewicz, Phys. Rev. C 71, 045502 (2005).

[50] C. J. Horowitz and J. Piekarewicz, Phys. Rev. Lett. 86, 5647 (2001); Phys. Rev. C 64, 062802 (2001).

[51] J. M. Lattimer and M. Prakash, Science 304, 536 (2004).

[52] J. M. Lattimer and M. Prakash, Phys. Rep. 442, 109 (2007), the Hans Bethe Centennial Volume 1906-2006.

[53] C. Ducoin, J. Margueron, C. Providência, and I. Vidaña, Phys. Rev. C 83, 045810 (2011).

[54] D. T. Loan, N. H. Tan, D. T. Khoa, and J. Margueron, Phys. Rev. C 83, 065809 (2011).

[55] M. Warda, B. Nerlo-Pomorska, and K. Pomorski, Nucl. Phys. A 635, 484 (1998).

[56] W.-Z. Jiang, B.-A. Li, and L.-W. Chen, Phys. Rev. C 76, 054314 (2007).
[57] M. Warda, X. Viñas, X. Roca-Maza, and M. Centelles, Phys. Rev. C 81, 054309 (2010).

[58] W. D. Myers and W. J. Świątecki, Nucl. Phys. A 336, 267 (1980).

[59] W. J. Świątecki, A. Trzcińska, and J. Jastrzȩbski, Phys. Rev. C 71, 047301 (2005).

[60] X. Viñas, M. Warda, M. Centelles, and X. Roca-Maza, Int. J. Mod. Phys. E 21, 1250029 (2012).

[61] S. Mizutori, J. Dobaczewski, G. A. Lalazissis, W. Nazarewicz, and P.-G. Reinhard, Phys. Rev. C 61, 044326 (2000).

[62] X. Roca-Maza, M. Centelles, F. Salvat, and X. Viñas, Phys. Rev. C 78, 044332 (2008).

[63] X. Roca-Maza, M. Centelles, F. Salvat, and X. Viñas, Phys. Rev. C 87, 014304 (2013).

[64] E. Chabanat, P. Bonche, P. Haensel, J. Meyer, and R. Schaeffer, Nucl. Phys. A 635, 231 (1998); 643, 441 (1998).

[65] J. Erler, N. Birge, M. Kortelainen, W. Nazarewicz, E. Olsen, A. M. Perhac, and M. Stoitsov, Nature 486, 509 (2012).

[66] M. V. Stoitsov, J. Dobaczewski, W. Nazarewicz, S. Pittel, and D. J. Dean, Phys. Rev. C 68, 054312 (2003).

[67] M. Del Estal, M. Centelles, X. Viñas, and S. K. Patra, Phys. Rev. C 63, 044321 (2001).

[68] R. W. Hasse and W. D. Myers, Geometrical Relationships of Macroscopic Nuclear Physics (Springer-Verlag, Heidelberg, 1988).

[69] E. Friedman, A. Gal, and J. Mares, Nucl. Phys. A 761, 283 (2005).

[70] E. Friedman, Hyperfine Interact. 193, 33 (2009).

[71] W. D. Myers and W. J. Świa̧tecki, Ann. Phys. (NY) 55, 395 (1969); 84, 186 (1974).

[72] W. D. Myers, Droplet Model of Atomic Nuclei (Plenum, New York, 1977).

[73] N. Schunck and J. L. Egido, Phys. Rev. C 78, 064305 (2008).

[74] A. Baran, K. Pomorski, and M. Warda, Z. Phys. A 357, 33 (1997). 